ARTICLE

Received 23 Apr 2014 | Accepted 30 Oct 2014 | Published 15 Dec $2014 \quad$ DOI: 10.1038/ncomms6712

\title{
Low MITF/AXL ratio predicts early resistance to multiple targeted drugs in melanoma
}

Judith Müller ${ }^{1}$, Oscar Krijgsman ${ }^{1}$, Jennifer Tsoi ${ }^{2}$, Lidia Robert ${ }^{3}$, Willy Hugo ${ }^{3}$, Chunying Song ${ }^{3}$, Xiangju Kong ${ }^{3}$, Patricia A. Possik¹, Paulien D.M. Cornelissen-Steijger ${ }^{1}$, Marnix H. Geukes Foppen ${ }^{4}$, Kristel Kemper ${ }^{1}$, Colin R. Goding 5 , Ultan McDermott ${ }^{6}$, Christian Blank ${ }^{4}$, John Haanen ${ }^{4}$, Thomas G. Graeber $2,7,8$, Antoni Ribas $2,3,8$, Roger S. Lo $0^{2,3,8} \&$ Daniel S. Peeper ${ }^{1}$

Increased expression of the Microphthalmia-associated transcription factor (MITF) contributes to melanoma progression and resistance to BRAF pathway inhibition. Here we show that the lack of MITF is associated with more severe resistance to a range of inhibitors, while its presence is required for robust drug responses. Both in primary and acquired resistance, MITF levels inversely correlate with the expression of several activated receptor tyrosine kinases, most frequently AXL. The MITF-low/AXL-high/drug-resistance phenotype is common among mutant BRAF and NRAS melanoma cell lines. The dichotomous behaviour of MITF in drug response is corroborated in vemurafenib-resistant biopsies, including MITF-high and -low clones in a relapsed patient. Furthermore, drug cocktails containing AXL inhibitor enhance melanoma cell elimination by BRAF or ERK inhibition. Our results demonstrate that a low MITF/AXL ratio predicts early resistance to multiple targeted drugs, and warrant clinical validation of $A X L$ inhibitors to combat resistance of BRAF and NRAS mutant MITF-low melanomas.

\footnotetext{
${ }^{1}$ Division of Molecular Oncology, The Netherlands Cancer Institute, Plesmanlaan 121, 1066CX Amsterdam, The Netherlands. ${ }^{2}$ Division of Molecular and Medical Pharmacology, David Geffen School of Medicine at University of California, University of California, Los Angeles (UCLA), 10833 Le Conte Avenue, Los Angeles, California 90095-1750, USA. ${ }^{3}$ Department of Medicine, David Geffen School of Medicine at University of California, Los Angeles (UCLA), 10833 Le Conte Avenue, Los Angeles, California 90095-7227, USA. ${ }^{4}$ Division of Medical Oncology, The Netherlands Cancer Institute, Plesmanlaan 121, 1066CX Amsterdam, The Netherlands. ${ }^{5}$ Ludwig Institute for Cancer Research, Nuffield Department of Clinical Medicine, University of Oxford, Old Road Campus Research Building, Headington, Oxford OX3 7DQ, UK. ${ }^{6}$ Wellcome Trust Sanger Institute, Genome Campus, Hinxton, Cambridge CB10 1SA, UK. 7 UCLA Metabolomics Center, Crump Institute for Molecular Imaging, California Nanosystems Institute, UCLA, 570 Westwood Plaza, Building 114, Los Angeles, California 90095-7227, USA. ${ }^{8}$ Jonsson Comprehensive Cancer Center (JCCC), 8-684 Factor Building, Los Angeles, California 90095-1781, USA. Correspondence and requests for materials should be addressed to D.S.P. (email: d.peeper@nki.nl).
} 
T he discovery of the activating $\mathrm{BRAF}^{\mathrm{V} 600 \mathrm{E}}$ mutation in roughly half of the melanomas ${ }^{1}$ has spurred the development of targeted therapies, which are associated with unprecedented clinical benefits. The small-molecule inhibitor vemurafenib, specifically targeting the mutant $\mathrm{BRAF}^{\mathrm{V} 600 \mathrm{E}}$ kinase, was the first standard of care for patients diagnosed with mutant BRAF metastatic melanoma ${ }^{2-4}$. Although this compound initially reduces tumour burden dramatically, eventually melanomas become resistant and tumours progress while on treatment ${ }^{5}$. Resistance to this treatment occurs by acquisition of additional mutations or other alterations that affect the mitogen-activated protein kinase (MAPK) pathway by either direct ${ }^{6-8}$ or indirect signalling ${ }^{6,9-11}$. Many resistance mechanisms somehow lead to reactivation of extracellular signal-regulated kinase (ERK), thereby restoring signalling of the oncogenic BRAF/MEK/ERK pathway ${ }^{12}$. In addition, PI3K pathway activation contributes to resistance to BRAF inhibition ${ }^{13}$. Less frequent but equally important to the phenomenon of targeted drug resistance is the observation that $\sim 15-20 \%$ of BRAF mutant melanoma patients fail to respond to BRAF inhibition already early on treatment, owing to intrinsic resistance. These patients have little therapeutic options, unless immunotherapy can be given ${ }^{14,15}$.

On the basis of the frequent occurrence of MAPK pathway reactivation, causing resistance to BRAF inhibition, the clinical rationale arose for combined treatment of BRAF and MEK inhibitors. In a phase $1 / 2$ clinical trial, the median progressionfree survival by the BRAF inhibitor dabrafenib and the MEK inhibitor trametinib was extended from 5.8 months on dabrafenib monotherapy to 9.4 months $^{16}$. However, also resistance to the combinatorial therapy eventually develops, leading to rapid disease recurrence.

Recently, an ERK inhibitor (SCH772984) with a dual mechanism of action was developed. It inhibits the enzymatic activity of ERK as well as its phosphorylation, and hence activation, by MEK $^{17}$. SCH772984 effectively blocks the proliferation of BRAF and BRAF/MEK inhibitor-resistant cells and has therefore been proposed as a new line of treatment for BRAF mutant (resistant) melanoma.

Despite its promise, we considered it conceivable that melanomas will eventually also overcome the cytotoxicity mediated by ERK inhibition. Therefore, we performed a gainof-function insertional mutagenesis screen to identify possible resistance mechanisms towards ERK inhibition. We identified an insertion in the MITF (Microphthalmia-associated transcription factor) locus, causing sharp upregulation of the corresponding master lineage transcription factor. MITF is responsible for pigmentation and indispensable for the development of the melanocytic lineage ${ }^{18}$. Its expression is usually maintained in melanoma, although MITF-negative specimens exist ${ }^{19}$.

The role of MITF in melanoma development and progression is equivocal. For example, high levels of MITF have been reported to block proliferation by the upregulation of cell cycle inhibitors $^{20,21}$. In seeming contrast, MITF was found to be amplified in $15 \%$ of metastatic melanomas, conceivably reflecting its oncogenic role ${ }^{22}$. Moreover, cells negative for MITF are known to display invasive properties ${ }^{19}$. In an attempt to reconcile these findings, a rheostat model has been proposed ${ }^{19}$. This pieces together three different phenotypes of melanoma cells that are dependent on MITF expression, ranging from differentiation (high MITF), proliferation (moderate MITF) and invasion (low MITF).

Our finding that increased MITF expression causes resistance to ERK inhibition is consistent with a recent report showing that MITF is sufficient to render melanoma cells resistant to MEK or ERK inhibitor-induced cell death ${ }^{9,23}$. However, those results do not speak to several seemingly opposite functions that have also been attributed to MITF. Therefore, we report here a more indepth study in melanoma cell lines and clinical specimens to investigate the contribution of MITF expression to the response of melanomas to clinically relevant inhibitors.

\section{Results}

Overexpressed MITF protects cells against ERK inhibition. To identify proteins conferring resistance to MAPK pathway inhibition, we used the recently available ERK inhibitor SCH772984 (ref. 17) in a lentiviral Validation-Based Insertional Mutagenesis (VBIM) screen system ${ }^{24}$. Carrying a green fluorescent proteinsequence and a strong CMV promoter, this virus integrates randomly into the genome, resulting in the activation of downstream sequences. This vector comes in three variants to integrate in the three possible open reading frames. Successful integrations lead to the overexpression of FLAG-tagged proteins and can be excised by 4-hydroxytamoxifen (4-OHT)-induced activation of Cre.

We used a low-passage human $\mathrm{BRAF}^{\mathrm{V} 600 \mathrm{E}}$ mutant melanoma cell line (04.07), which is intermediately sensitive to SCH772984 (not all cells are killed by the ERK inhibitor even when used at a high concentration; Supplementary Fig. 1A). We infected this cell line with the three versions of the insertional mutagenesis vector (SD1-3; Supplementary Fig. 1B). After 3 weeks of culturing in the

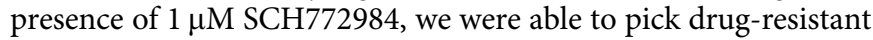
clones, which were individually expanded. We first confirmed that the resistance occurred owing to an insertion, providing a selective and reversible advantage in the presence of ERK inhibition, as resistance could be reverted by 4-OHT-treatment (Supplementary Fig. 1C). In the majority of the clones, we detected one or more Flag-tagged proteins, indicating that successful in-frame insertions had occurred into the genome, leading to overexpression of fusion proteins (Fig. 1a).

We observed an $\sim 55 \mathrm{kDa}$ FLAG-tagged protein in multiple independent clones, raising the possibility that a common gene was activated. Using splinkerette PCR followed by Sanger sequencing, we identified an insertion in intron 2-3 of the MITF gene locus (Supplementary Fig. 1D). This finding was confirmed by qPCR, showing up to 100 -fold overexpression of MITF, and immunoblotting of six individual clones (Fig. $1 \mathrm{~b}$ and Supplementary Fig. 1E).

To functionally validate if MITF can induce resistance to ERK inhibition, we overexpressed it in a panel of melanoma cell lines using a lentiviral system. Exogenous overexpression of MITF promoted the survival and proliferation in three independent $\mathrm{BRAF}^{\mathrm{V} 600 \mathrm{E}}$ melanoma cells lines in the presence of ERK inhibitor (Fig. 1c), despite complete ERK-pathway inhibition (Fig. 1d). The same protective effect of MITF was seen for the preclinical BRAF inhibitor PLX4720 or a MEK inhibitor (trametinib or GSK1120212; Supplementary Fig. 1F). These observations are in agreement with, and extend, recent findings on the role of MITF in the response to MEK and ERK inhibitors ${ }^{9,23}$.

MITF loss occurs in acquired resistance in vitro and in vivo. To determine the regulation of MITF expression during acquired resistance, we generated several cell lines that are resistant to $1 \mu \mathrm{M}$ SCH772984 (Supplementary Fig. 2A) or $3 \mu \mathrm{M}$ PLX4720 (Fig. 2a and Supplementary Fig. 2B) by culturing under increasing drug exposure. In line with our findings from the VBIM screen, we observed either the maintenance of high MITF expression or a moderate increase in MITF expression in three out of seven PLX4720-resistant lines (888mel, A375, D10; Fig. 2b), along with increased expression of several MITF targets and more pigmentation of the cells. 
a

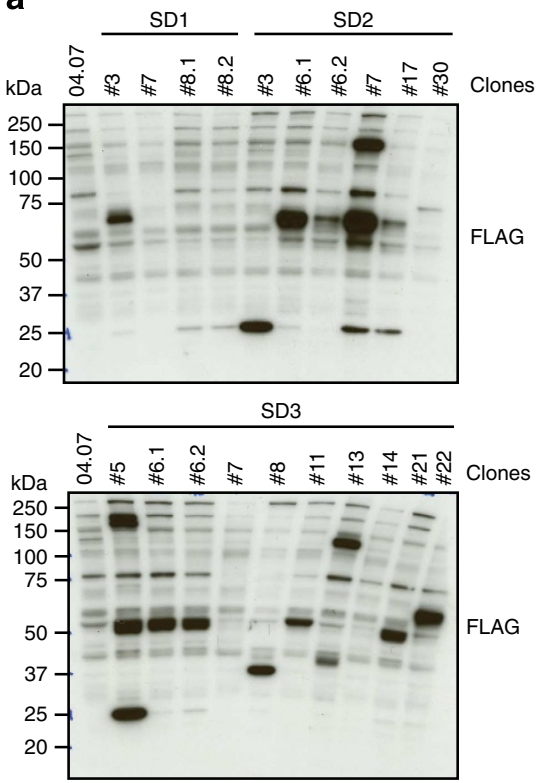

b

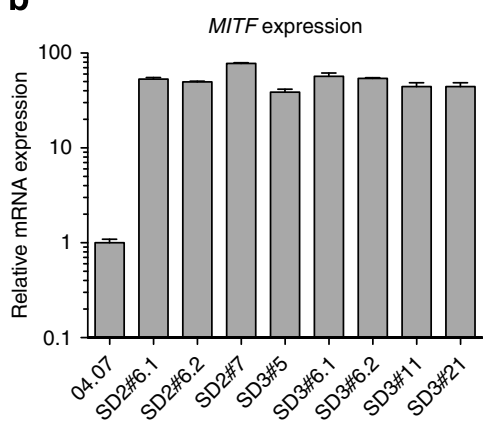

C

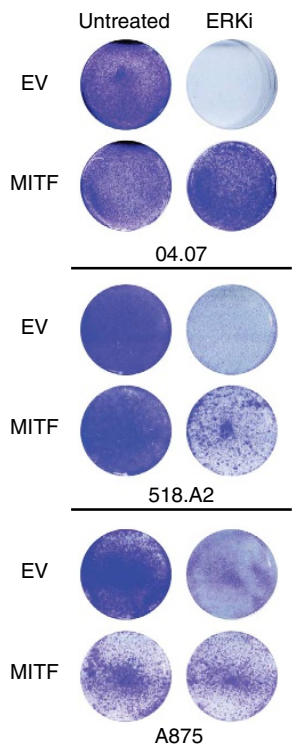

d

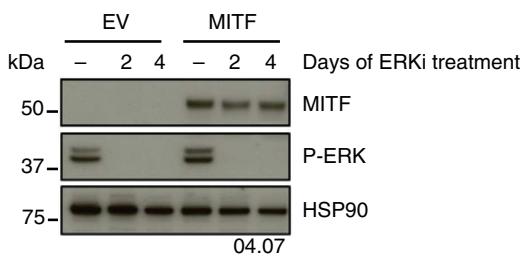

Figure 1 | Overexpressed MITF protects cells against ERK inhibition. (a) An insertional mutagenesis screen was performed using the VBIM system. A BRAF mutant melanoma cell line of low passage (04.07) was infected with three VBIM vectors (SD1-3; to cover the three reading frames) and treated with $1 \mu \mathrm{M}$ of the ERK inhibitor SCH772984. Drug-resistant clones were established and analysed for FLAG-tagged proteins resulting from the integration of the viral cassettes. (b) Integration of the VBIM cassettes resulted in MITF overexpression in six independent ERKi-resistant clones, as shown by qRT-PCR, compared with parental 04.07 cells. Note that only clones derived from separate plates were considered as independent clones. Error bars denote s.d. for technical replicates. (c) Lentiviral overexpression of MITF resulted in increased resistance to long-term ERK inhibition in three independent BRAF mutant melanoma cell lines as shown by crystal violet staining. (d) Immunoblot of 04.07 cells confirmed MITF overexpression and inactivation of ERK after inhibitor treatment. HSP9O served as a loading control.

In contrast, four out of seven PLX4720-resistant lines (SkMel28, Colo679, WM266-4 and 93.03) had downregulated MITF expression to almost undetectable levels relative to their parental counterparts (Fig. 2b). In almost all resistant cell lines, ERK and RSK were reactivated to the levels seen in their untreated counterparts. Similarly, four out of six cell lines resistant to $1 \mu \mathrm{M}$ SCH772984 had decreased expression of MITF accompanied by decreased expression of several target genes (Supplementary Fig. 2A). The loss of MITF occurred at the transcriptional level; in fact, the MITF mRNA levels were undetectable, as measured by quantitative reverse transcriptionPCR (qRT-PCR) analysis (Fig. 2c). Thus, one set of acquired drug-resistant cell lines maintained or increased MITF expression; we call these MITF ${ }^{\text {acq_maint }}$ cells. In contrast, for at least half of the cell lines we observed the opposite: a sharp downregulation of MITF expression; we call these MITFacq_loss cells. The differential regulation of MITF during the acquisition of drug resistance suggests that there are two classes of MITF-expressing melanoma cell lines, which differ in their response to MAPK pathway inhibition: in MITFacq_maint cells, MITF signalling is maintained or even induced, potentially contributing to drug resistance. In MITF ${ }^{\text {acq_loss }}$ cells, in which MITF is downregulated, maintenance of MITF expression is not required for drug resistance.

To study the dynamics of MITF downregulation in relation to the acquisition of a resistant phenotype, two independent MITF $^{\text {acq_loss }}$ melanoma cell lines from an independent panel (M229 and M238; ref. 13) were permanently exposed to $1 \mu \mathrm{M}$ vemurafenib and monitored over time. MITF expression sharply dropped on short-term exposure to PLX4720 and was further decreased in the remaining drug-tolerant population, drugtolerant proliferating population and in resistant cells (Supplementary Fig. 2C,D). These results show that acquired resistance is accompanied by a rapid decrease in MITF, and confirm that this is regulated at the transcriptional level.

To corroborate these in vitro findings in a physiologically more relevant setting, we performed immunohistochemical staining for MITF on a limited set of human melanoma biopsies. The samples were taken from patients before treatment with vemurafenib and after patients had relapsed with vemurafenib-resistant tumours. 
a

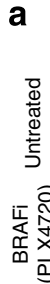<smiles>[R10]C1CC1</smiles>
sensitive resistant

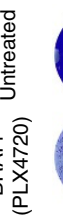
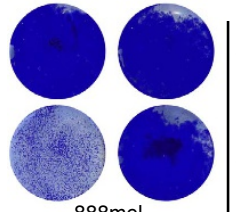

PLX PLX sensitive resistan

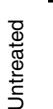

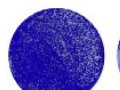

这

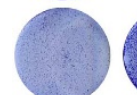

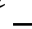

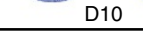

10

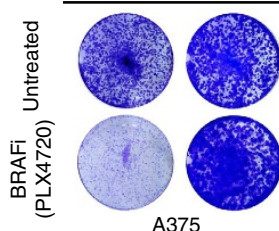

A375

C

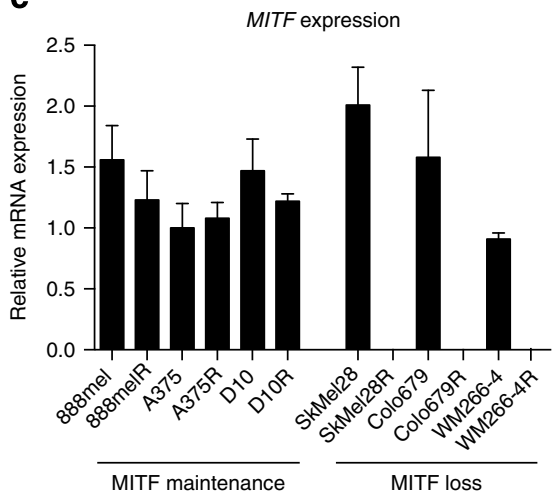

e

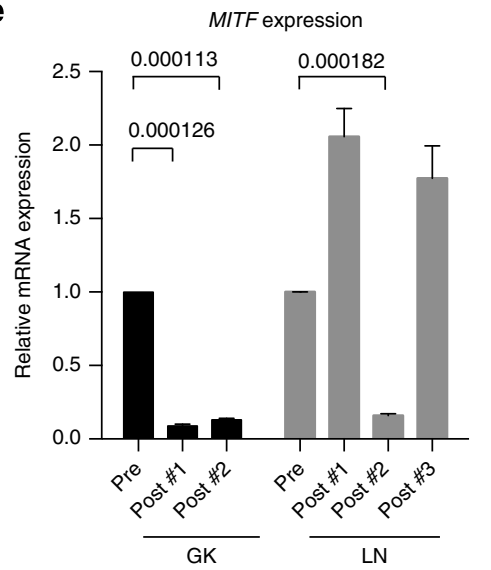

b

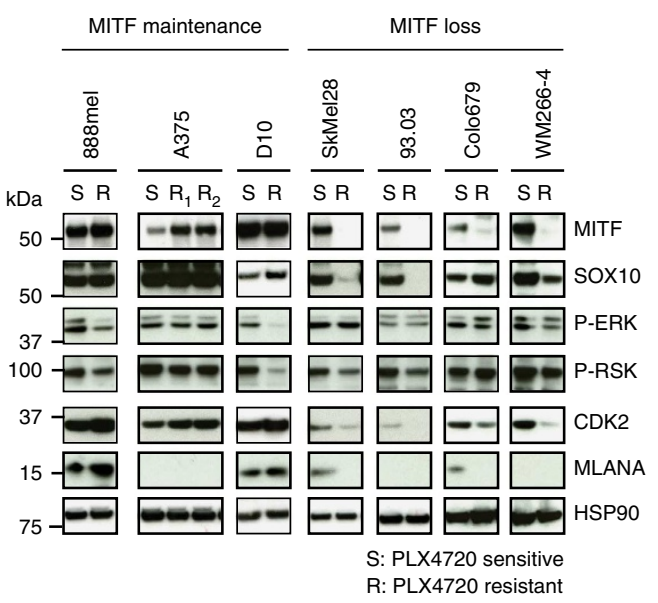

d

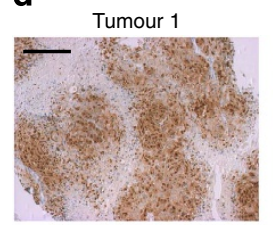

Tumour 2
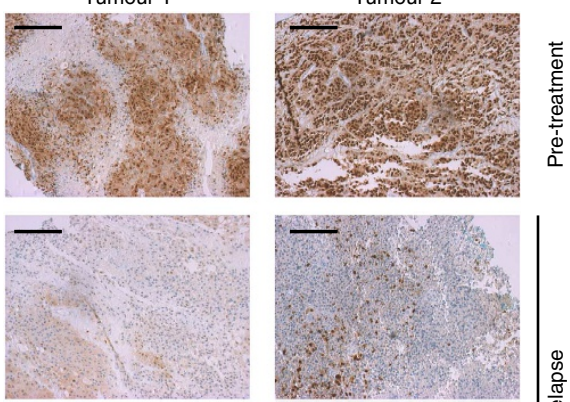

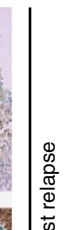

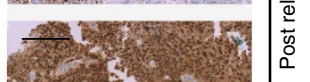

\section{$\stackrel{\vec{\omega}}{E}$}

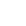


In one out of four matched (pre-treatment and post-relapse) sample sets, we observed a sharp drop in MITF abundance (Fig. 2d, tumour 1). For another patient, we observed a remarkable differential response: while there was abundant expression in the pre-treatment sample, one relapsed melanoma metastasis showed elevated levels of MITF, whereas another had lost detectable expression in most of the resistant tumour cells (Fig. 2d, tumour 2). These results are in agreement with our in vitro findings and indicate that melanoma drug resistance in vivo is associated not only with gain of MITF expression but also with its loss, even in different relapsed tumour clones from the same patient.

To validate these observations in an independent set of BRAF $^{\mathrm{V} 600 \mathrm{E}}$ mutant melanomas, we compared MITF mRNA levels before treatment and after resistance had occurred. Again, we found that MITF expression strongly decreased in several resistant tumour clones compared with their treatment-naïve counterparts (Fig. 2e). In this cohort of 12 patients who developed resistance to vemurafenib treatment, three relapsed with one or more tumours that significantly decreased in MITF expression (25\%). The entire cohort comprised 29 vemurafenib-resistant relapses, of which four displayed a significant downregulation of MITF at the mRNA level. In comparison, in five patients, tumours with elevated MITF expression were found. Thus, MITF $^{\text {acq_maint }}$ and MITF ${ }^{\text {acq_loss }}$ cells co-exist in vivo and the loss of MITF is commonly seen in the context of MAPK pathway inhibition, both in vitro and in vivo.

MITF loss causes cross-resistance to MAPK pathway inhibition. To further characterize the differences between the MITF $^{\text {acq_maint }}$ and MITF ${ }^{\text {acq_loss }}$ cells, we exposed them to several inhibitors of the MAPK pathway, either alone or in combination. PLX4720-resistant MITF ${ }^{\text {acq_maint }}$ cells were resistant to another BRAF inhibitor (dabrafenib) but were as responsive to MEK or ERK inhibition as their drug-sensitive counterparts (Fig. 3a, top). In contrast, PLX4720-resistant MITF ${ }^{\text {acq_loss }}$ cells displayed crossresistance to the full panel of MAPK pathway inhibitors, even when BRAF and MEK were inhibited simultaneously (Fig. 3a, bottom). Phosphorylation of ERK was similarly suppressed across all cell lines, demonstrating that in all cases the drugs were equally effective towards their respective targets (Fig. 3b). In

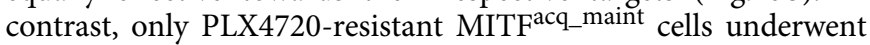
apoptosis on drug treatment as demonstrated by PARP cleavage, whereas MITF acq_loss cells did not display any sign of apoptosis. In a larger set of melanoma cell lines, comparing three MITF $^{\text {acq_maint }}$ and three MITF ${ }^{\text {acq_loss }}$ cell lines, we observed that the latter group tolerated exposure to higher drug concentrations

Figure 3 | MITF loss causes cross-resistance to MAPK pathway inhibition. (a) Two PLX4720-resistant cell lines (888mel and SkMel28) and their treatment-naïve counterparts were treated with either the ERKi SCH772984 $(0.5 \mu \mathrm{M})$, the MEKi trametinib $(0.3 \mu \mathrm{M})$, BRAF inhibitor dabrafenib $(3 \mu \mathrm{M})$ or a combination as indicated and stained with crystal violet after 6 days of treatment. (b) Immunoblotting for the indicated proteins confirmed the downregulation of the MAPK pathway (as judged by P-ERK) and showed cleaved PARP (arrow) as a measure of apoptosis of the cell lines used in a. CDK4 served as a loading control. (c) Six PLX4720resistant cell lines and their treatment-naïve counterparts were separated in groups that either lose or maintain (or increase) MITF expression on acquiring resistance. These cells were exposed to the MEKi trametinib or the ERKi SCH772984 in dose-response curves. IC40 (for MEKi) or IC50 (for ERKi) were calculated from the curves. Fold increase in IC40 or IC50 was determined comparing resistant to sensitive cells. Mean was calculated from three independent experiments, error bars indicate s.d. than MITF ${ }^{\text {acq_maint }}$ cells, and hence are more prone to develop cross-resistance (Fig. 3c, Supplementary Fig. 2G,H).

Loss of MITF is accompanied by increased invasiveness. In melanoma, low MITF expression has been associated with a phenotypic switch, including increased invasiveness ${ }^{19}$. To determine whether MITF ${ }^{\text {acq_loss }}$ cells show such properties, we

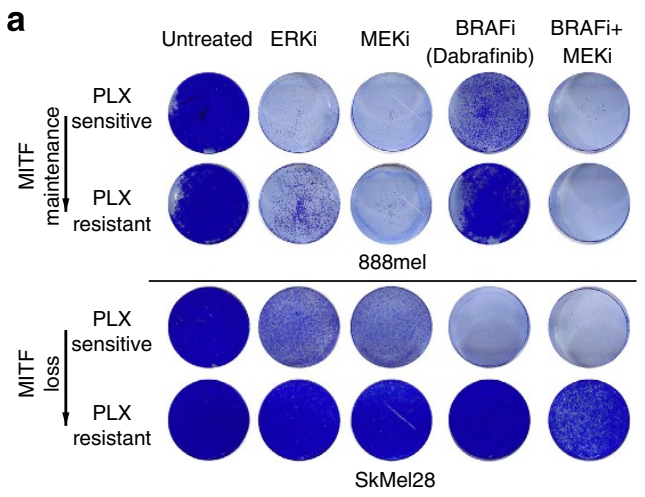

b

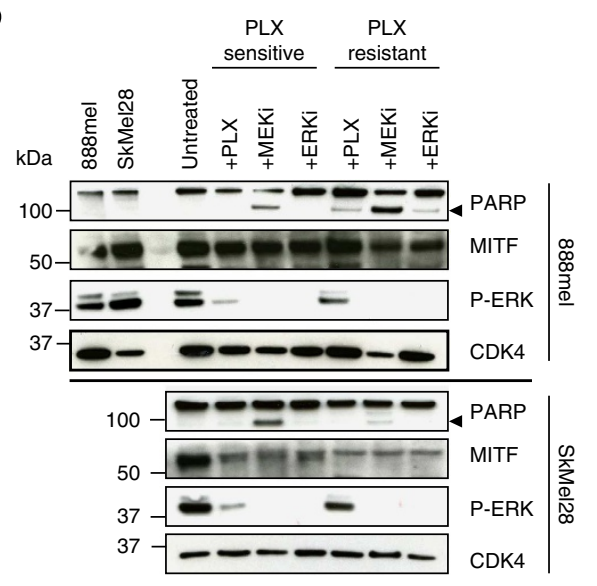

C
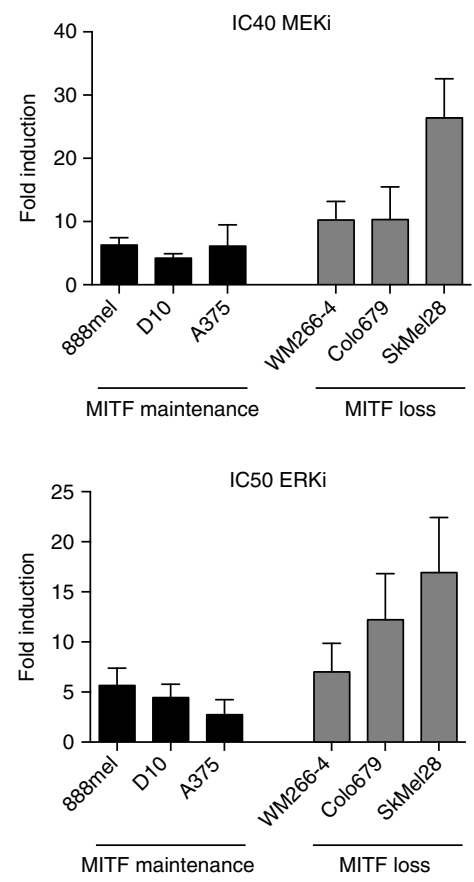
transferred them to a matrigel-coated chamber and monitored their invasive potential. Indeed, MITF ${ }^{\text {acq_loss }}$ cells were endowed with increased invasive capacity when compared to MITF ${ }^{\text {acq_maint }}$ cells (Fig. 4a,b). In MITF ${ }^{\text {acq_loss }}$ cell lines that expressed E-Cadherin before drug exposure, this epithelial protein was lost on the acquisition of drug resistance (Fig. 4c). In WM266-4 cells in which E-Cadherin protein expression was below detection level, a decrease in $C D H 1$ mRNA was detected by
qRT-PCR (Supplementary Fig. 2F). N-Cadherin was more abundant in MITF $^{\text {acq_loss }}$ cells already before treatment but remained unaltered after resistance occurred (Fig. $4 \mathrm{c}$ and Supplementary Fig. 2F). We further investigated additional epithelial to mesenchymal transition (EMT) markers, including the transcription factors $Z E B 1$ and $Z E B 2$, and found a remarkable downregulation in $Z E B 2$ expression in three MITF $^{\text {acq_loss }}$ cell lines and a concomitant increase of ZEB1 in two of the three cell a

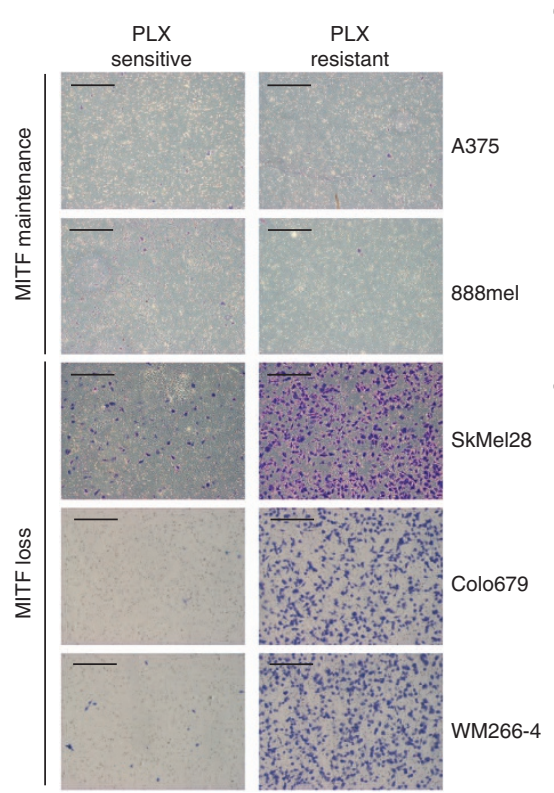

b
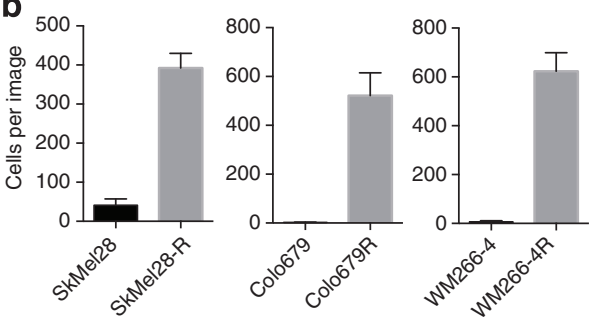

C

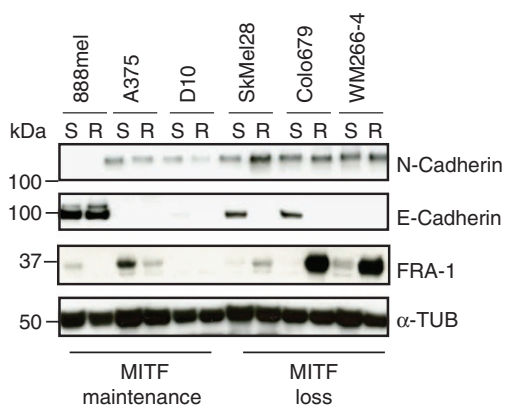

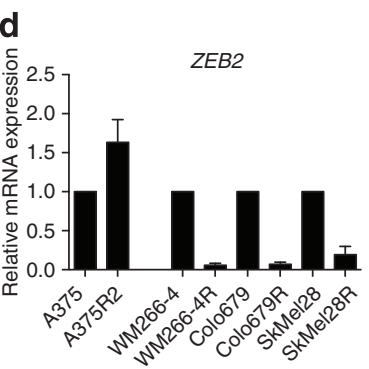

e

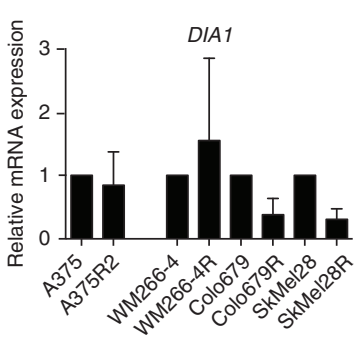

f

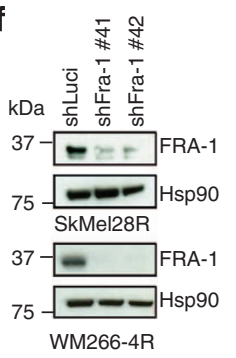

g

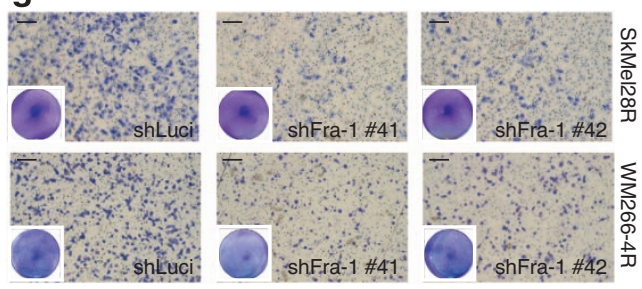

h

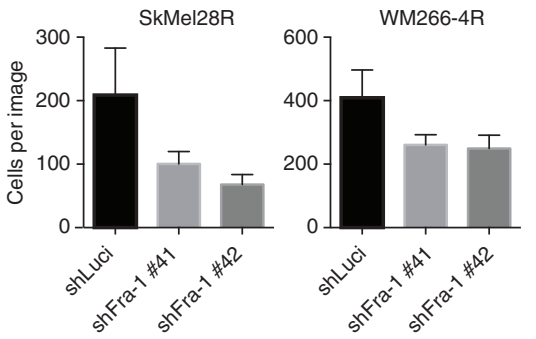

Figure 4 | Loss of MITF is accompanied by increased invasiveness. (a) PLX4720-resistant melanoma cells and their treatment-naïve counterparts were seeded on a matrigel-coated invasion chamber for $12 \mathrm{~h}$. Cells that invaded through the matrix were stained with crystal violet. Scale bars, $500 \mu \mathrm{m}$. (b) Quantification of a from at least five images. (c) PLX4720-resistant cells (R) and their treatment-naïve (S) counterparts were blotted for EMT markers. $\alpha$-Tubulin served as a loading control. (d) ZEB1 and ZEB2 mRNA were measured in acquired PLX4720-resistant cells and their treatment-naïve counterparts. The results are based on three independent experiments and normalized to RPL13. Data are shown as mean + s.d. (e) mRNA expression of Dia1 was measured in acquired PLX4720-resistant cells and their treatment-naïve counterparts. The results are based on three independent experiments and normalized to RPL13. Data are shown as mean + s.d. (f) Knockdown of Fra-1 in two PLX4720-resistant cell lines was documented on immunoblot. HSP90 served as a loading control. (g) Two PLX4720-resistant melanoma cells transduced with Fra-1 knockdown and control cells were seeded on a matrigel-coated invasion chamber for $12 \mathrm{~h}$. Cells that invaded through the matrix were stained with crystal violet. Inserts show proliferation in $12 \mathrm{~h}$ on polystyrene culture dishes. Scale bars, $200 \mu \mathrm{m}$. (h) Quantification of $\mathbf{g}$ of at least five images. 
lines (Fig. 4d). In WM266-4 cells, instead, TWIST mRNA was highly elevated in resistant cells compared with treatment-naïve ones, suggesting that it might compensate for ZEB1 in mediating EMT and invasion (Supplementary Fig. 2E). Further, Dia1 has been described to be downregulated in MITF-dependent invasion ${ }^{19}$. DIA1 mRNA transcription had dropped in two of three MITF acq_loss cell lines (Fig. 4e). In addition, the transcription factor Fra-1, which we recently demonstrated to be involved in invasion and metastasis ${ }^{25}$, was increased in all three MITFacq_loss cells (Fig. 4c). To determine if the resistanceinduced invasiveness was mediated by Fra-1, we introduced Fra-1 knockdown vectors into two MITF $F^{\text {acq_loss }}$ cell lines (Fig. 4f). The depletion of Fra-1 expression decreased the invasive capacity of these cells without affecting cell proliferation (Fig. 4g,h). Thus, in addition to displaying a MAPK pathway inhibition cross-resistant phenotype, MITFacq_loss cells are much more invasive than MITF ${ }^{\text {acq_maint }}$ cells, which occurs in a Fra-1-dependent manner, and they display properties of EMT.

Absence of MITF marks intrinsically insensitive cells. The above experiments focused on the regulation and role of MITF in the context of acquired targeted drug resistance. Next we investigated the impact of different levels of endogenous MITF expression on treatment-naïve melanoma cells regarding sensitivity to MAPK pathway inhibition. For that, we grouped BRAF mutant melanoma cells in 'MITF ${ }^{\text {endo_hi, }}$ and 'MITF ${ }^{\text {endo_lo' }}$ cells based on their endogenous protein expression levels (Fig. 5a) and transcript expression (Supplementary Fig. 3A). As expected, most MITF ${ }^{\text {endo_hi }}$ cell lines expressed MITF-target gene products like CDK2 and MelanA, whereas those proteins were expressed to lower or even undetectable levels in MITF ${ }^{\text {endo_lo }}$ cells.

We then determined the spectrum of drug sensitivity among a panel of MITF $F^{\text {endo_lo }}$ and MITF ${ }^{\text {endo_hi }}$ cells. On exposure to $5 \mu \mathrm{M}$ PLX4720 for 6 days, there was a clear survival benefit of MITF ${ }^{\text {endo_lo }}$ cells, as illustrated by viability assays and PARP cleavage (Fig. 5b,c) This difference was not caused by insufficient pathway inhibition since ERK phosphorylation was diminished irrespective of MITF status. In agreement, comparing four MITF $^{\text {endo_hi }}$ with four MITF ${ }^{\text {endo_lo }}$ cell lines, the latter showed a dramatic increase in resistance to MEKi as was illustrated by an up to 1,000 -fold increase in IC50 values (Fig. 5d and Supplementary Fig. 3B). This phenomenon was also seen on combinational inhibition of BRAF and MEK, indicating a much more robust drug resistance phenotype of MITF ${ }^{\text {endo }}{ }^{10}$ cells than MITF ${ }^{\text {endo_hi }}$ cells (Fig. 5e).

Seeking whether these findings could be confirmed in an independent data set, we investigated MITF expression and drug sensitivity in an additional panel of human $\mathrm{BRAF}^{\mathrm{V} 600 \mathrm{E}}$ mutant melanoma cell lines. Again we observed an inverse correlation between endogenous MITF expression and the IC50 values for vemurafenib and ERK inhibitor treatment (Fig. 5f).

AXL and other RTKs are inversely correlated with MITF. To identify proteins that are responsible for protecting MITFendo_lo cells from MAPK pathway inhibition, we performed next-generation RNA sequencing on three MITF ${ }^{\text {endo_lo }}$ and three MITF $^{\text {endo_hi }}$ BRAF ${ }^{\mathrm{V} 600 \mathrm{E}}$ cell lines. We found that several receptor tyrosine kinases (RTKs) were expressed to higher levels in the MITF ${ }^{\text {endo_lo }}$ cell lines, including AXL, epidermal growth factor receptor (EGFR) and platelet-derived growth factor receptor (PDGFR $\beta$; Fig. 6a and Supplementary Fig. 4A). Encouraged by these findings, we compared the phosphorylation status of RTKs in an MITF $F^{\text {endo_lo }}$ and an MITF $F^{\text {endo_hi }}$ cell line. Already in the untreated control, a subset of RTKs was activated in MITF endo_lo cells (Fig. 6b, upper array). Exposure for 2 days to $5 \mu \mathrm{M}$ PLX4720 further increased the activation of several RTKs in MITF ${ }^{\text {endo_lo }}$ cells, whereas only a minor activation of RTKs could be detected in MITF ${ }^{\text {endo_hi }}$ cells (Fig. 6b, lower array). In addition to activation, we observed increased expression levels of particularly AXL, PDGFR $\beta$ and EGFR in MITF ${ }^{\text {endo_lo }}$ cells compared with MITF $^{\text {endo_hi }}$ cells in a larger set of melanoma cell lines (Fig. 6c). In an independent data set (from Fig. 5f), we correlated MITF and AXL mRNA expression and observed a significant inverse correlation (correlation coefficient of -0.84 and correlation $P$-value of $7 \mathrm{E}^{-09}$; Supplementary Fig. $\left.4 \mathrm{~B}\right)$. In addition, this data set validated the increased resistance to BRAF and ERK inhibition in MITF-low/AXL-high cells. To confirm these findings in vivo, we extracted protein from patient-derived xenografts (PDX)-derived tumour samples and blotted for MITF, MITF targets and RTKs. Similar to our in vitro findings, AXL and PDGFR $\beta$ were only expressed in tumours where MITF was absent or expressed at very low levels (Fig. 6d). Consistent with these data, analysis of 356 TCGA melanoma transcriptomes supported the anticorrelation between MITF and RTKs including AXL (Fig. 6e).

RTK inhibition increases effect of BRAF inhibition. These results raised the possibility that high expression of one or more RTKs contributed to the intrinsically resistant phenotype of MITF ${ }^{\text {endo_lo }}$ cells. This prompted us to expose melanoma cells to compounds specifically targeting the RTKs AXL, EGFR and PDGFR $\beta$, either alone or in combination. Single inhibition of either AXL or EGFR led to melanoma cell death only at high drug concentration irrespective of their MITF expression (Supplementary Fig. 4C). In contrast, the combined treatment of single RTK inhibition with MAPK pathway inhibitors caused a considerable decrease in viability (Fig. 7a,b). In a triple combination, treatment of MITF endo_lo cells with AXL, EGFR and PDGFR inhibitors further sensitized MITF ${ }^{\text {endo_lo }}$ cells to BRAF inhibition alone or combined BRAF + MEK inhibition (Fig. 7a,b, Supplementary Fig. 5A,B). These results confirm that MITF $^{\text {endo_lo }}$ cells are highly intrinsically drug resistant. However, at the same time, these data indicate that several RTKs, including AXL, contribute to drug resistance.

Next we determined the expression pattern of these RTKs in the setting of acquired resistance, particularly in PLX4720-sensitive cells and their resistant counterparts. All three MITF ${ }^{\text {acq_loss }}$ cell lines examined, which had lost MITF expression after prolonged exposure to PLX4720, showed a strong upregulation of AXL, whereas PDGFR $\beta$ and EGFR were upregulated in only one of these cell lines (Fig. 7c). Furthermore, MITFFacq_loss cells displayed increased phosphorylation of these RTKs, which was not detected before drug resistance was acquired (Fig. 7d). Together these results suggest that increased expression and activity of a set of RTKs, particularly AXL, is seen not only in innate but also acquired resistance of melanoma cells. Consistently, in a matched set of pre-treatment and post-relapse samples, the MITF/AXL anticorrelation was recapitulated (Fig. 7e).

To determine whether AXL inhibition can be used to impact on $\mathrm{BRAF}^{\mathrm{V} 600 \mathrm{E}}$ mutant melanoma cells resistant to MAPK pathway inhibition, we exposed AXL-expressing PLX4720resistant cells and their treatment-naïve counterparts to the AXL inhibitor R428. In two of the three resistant cell lines we observed a profound, and in one a modest, decrease in viability on drug treatment (Fig. 7f). Taken together, these data indicate that AXL is a key determinant of resistance of melanomas to MAPK pathway inhibition; targeting this RTK in the context of combination therapy is likely to impact on innate or acquired resistant melanomas in the setting of MAPK pathway inhibition.

MITF is a predictive biomarker also in NRAS mutant melanomas. Finally, to extend our findings to another relevant melanoma 
a

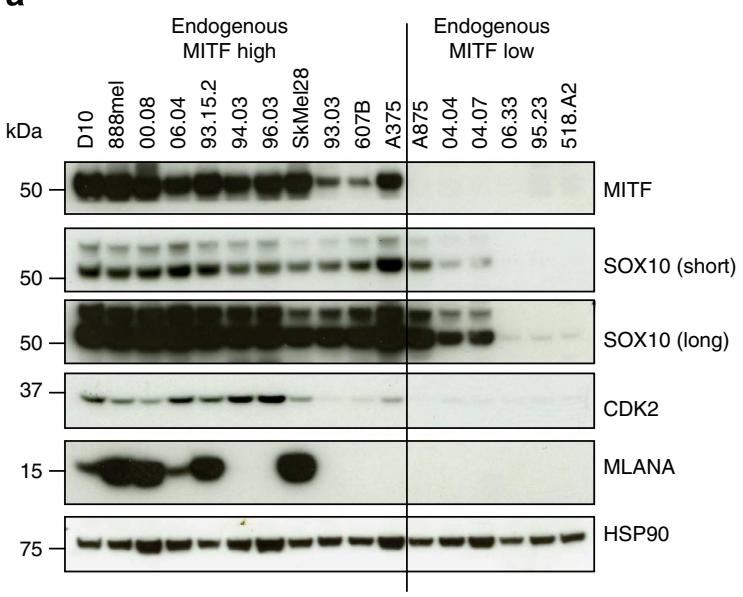

b

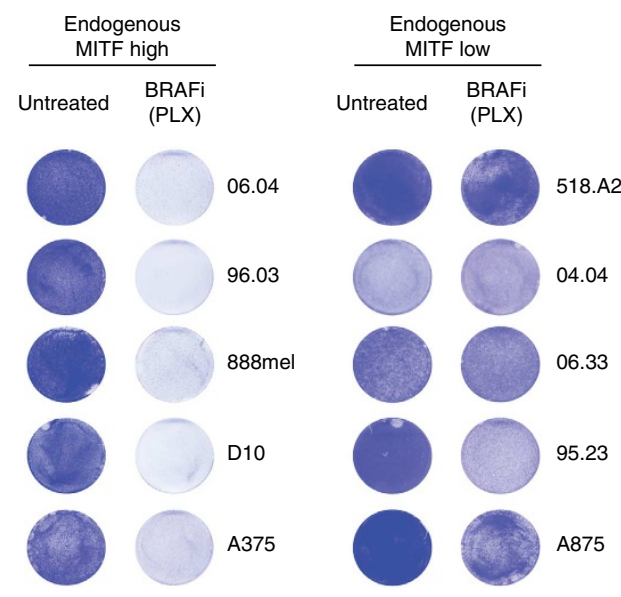

C

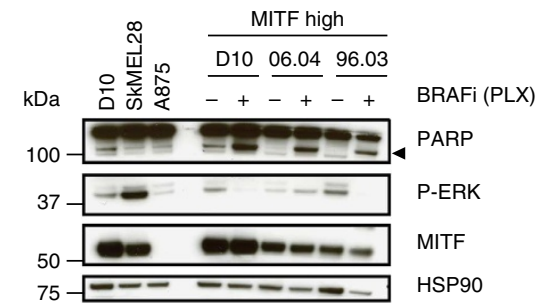

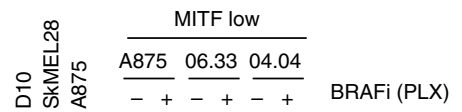

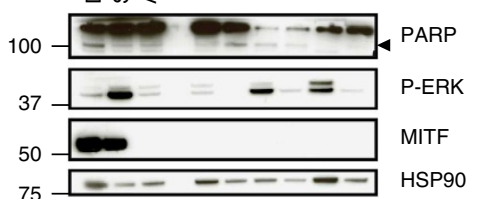

d

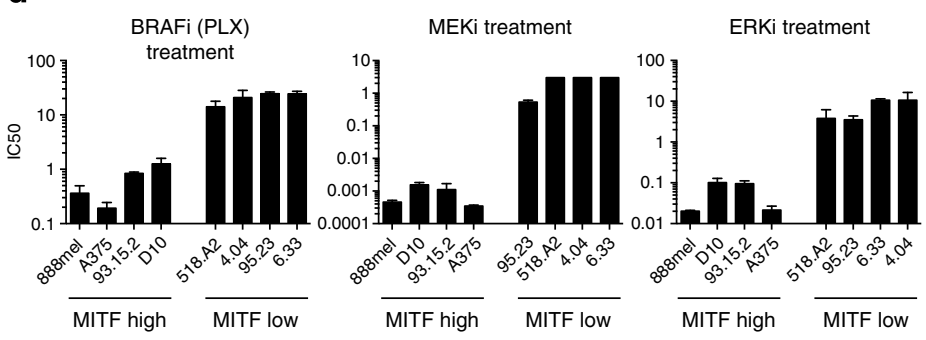

e
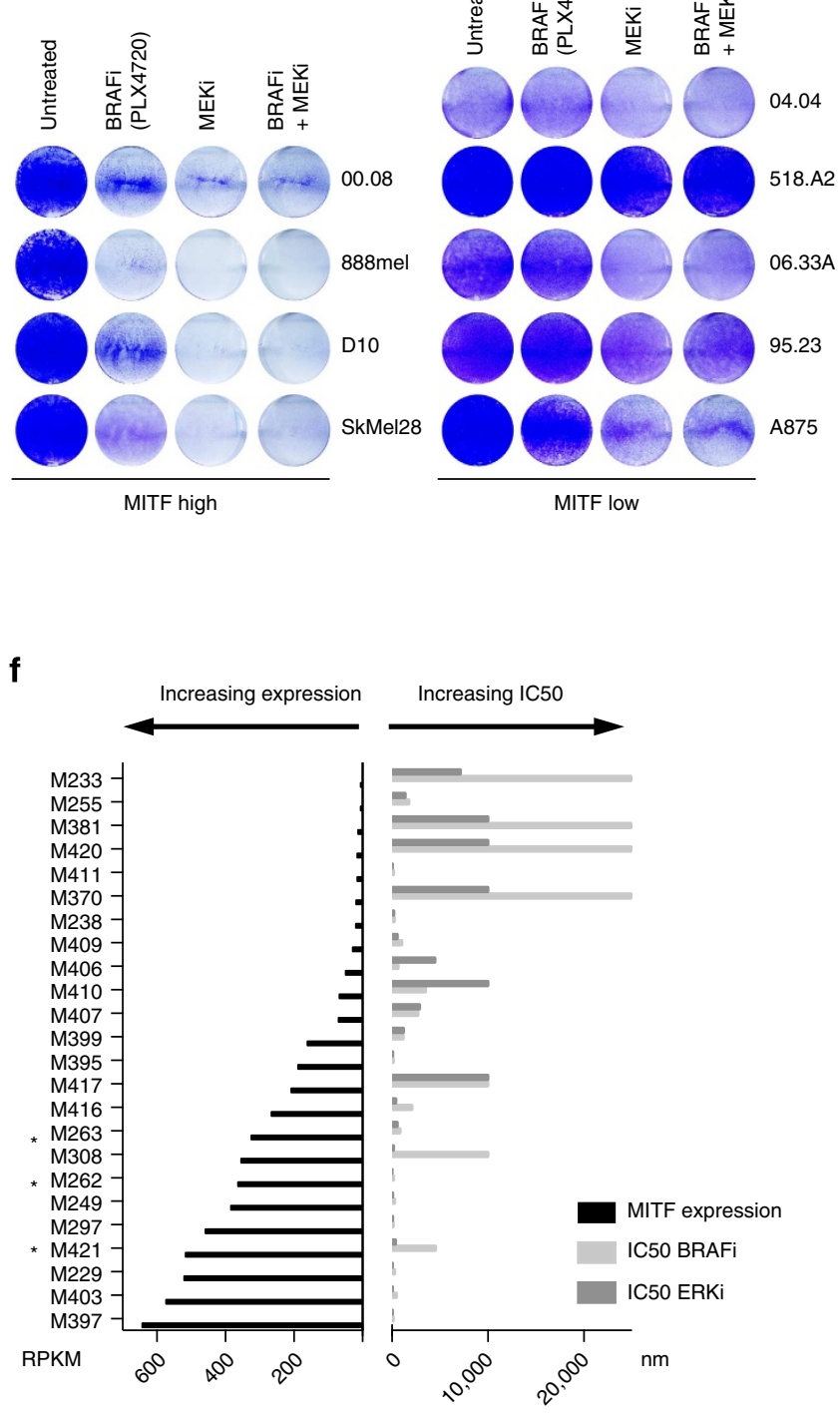

f

Figure 5 | Absence of MITF marks intrinsically insensitive cells. (a) Treatment-naïve BRAF mutant melanoma cells were grouped based on their MITF expression by immunoblotting, also for additional proteins as indicated. HSP90 served as a loading control. (b) BRAF mutant melanoma cells were plated at low density and treated with $5 \mu \mathrm{M}$ PLX4720 for 6 days or left untreated and stained with crystal violet. (c) For a subset of the cells lines from b, MAPK pathway inhibition and apoptosis indicated by cleaved PARP (arrow) after 3 days of treatment with $5 \mu$ M PLX4720 was confirmed by immunoblotting. HSP90 served as a loading control. (d) Sensitivity of MITFendo_hi and MITFendo_lo treatment-naïve melanoma cell lines to the BRAFi PLX4720, to the ERKi SCH772984 and to the MEKi trametinib was determined in dose-response curves. Mean was calculated from three independent experiments, error bars indicate s.d. (e) MITF endo_hi and MITF endo_lo BRAF $\mathrm{V} 600 \mathrm{E}$ mutant melanoma cell lines were plated at low density and treated with either BRAFi $(2 \mu \mathrm{M})$, MEKi trametinib $(0.1 \mu \mathrm{M})$ or a combination. After 6 days, cells were stained with crystal violet. (f) An independent set of treatment-naïve BRAF ${ }^{6} 60 \mathrm{E}$ mutant melanoma cell lines was grouped based on MITF expression, and resistance to the BRAFi (vemurafenib) and the ERKi $\mathrm{SCH} 772984$ was determined by dose-response curves. Cell lines with MITF amplification are marked with an asterix. 
a

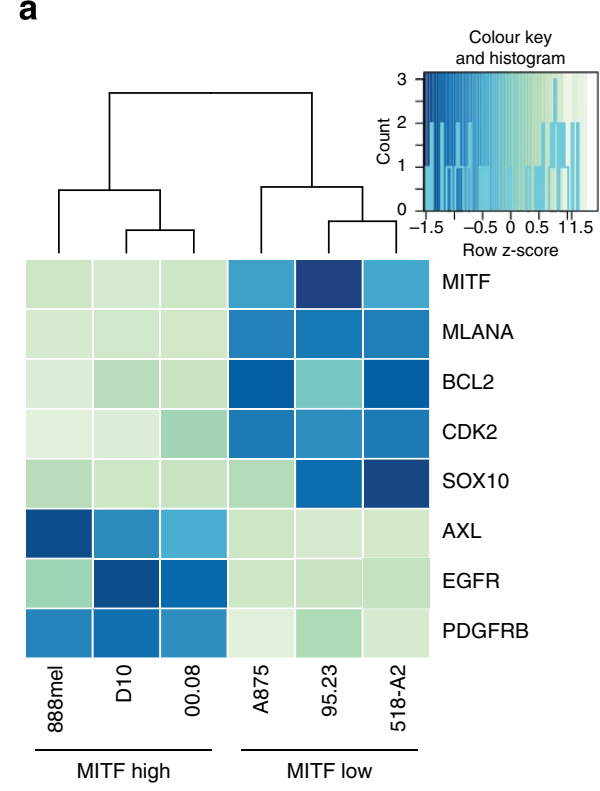

b
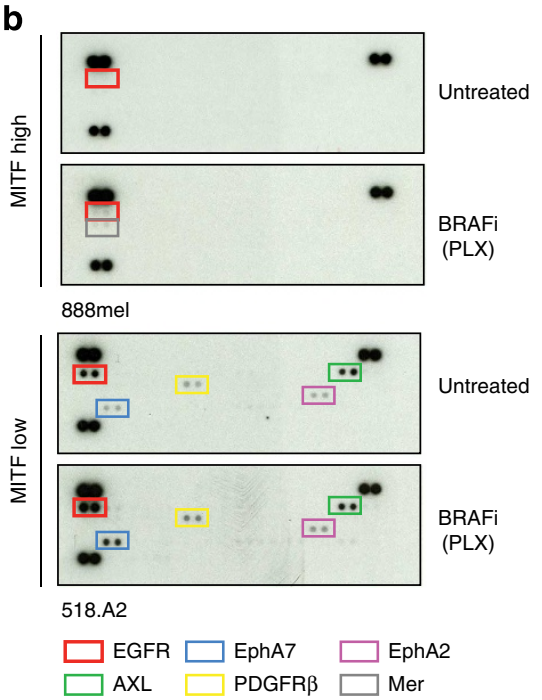

C

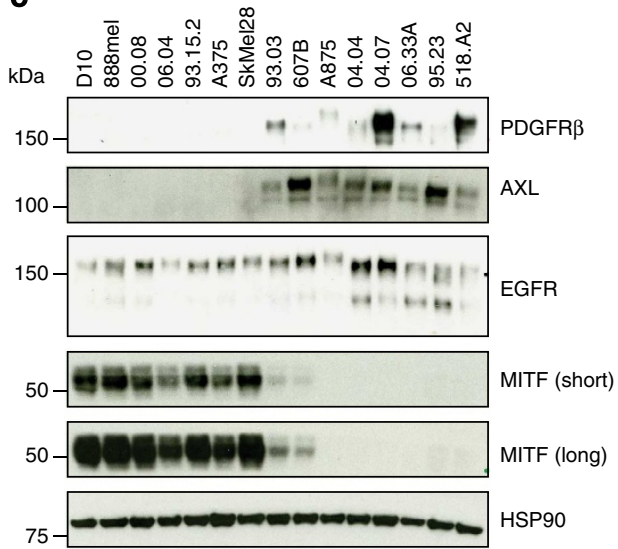

d

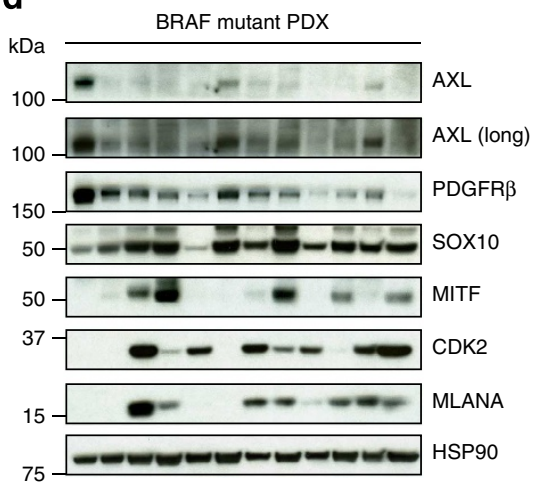

e

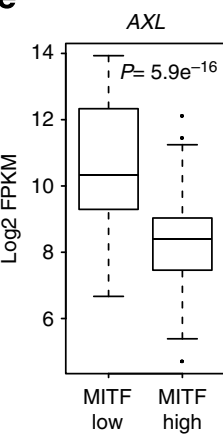

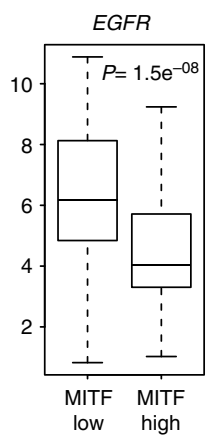

Figure 6 | AXL and other RTKs are inversely correlated with MITF. (a) RNA sequencing was performed in three MITFendo_hi and three MITFendo_lo $\mathrm{BRAF}^{\mathrm{V} 600 \mathrm{E}}$ mutant melanoma cell lines. The genes in the heatmap were selected based on the MITF expression, expression of MITF-target genes and expression of the RTKs AXL, EGFR and PDGFR $\beta$. (b) A phospho-RTK array was performed comparing one MITFendo_hi and one MITFendo_lo melanoma cell line untreated or treated with $5 \mu \mathrm{M}$ PLX4720 for 2 days. (c) MITFendo_hi and MITFendo_lo cell lines were immunoblotted for the RTKs PDGFR $\beta$, AXL and EGFR. HSP90 served as a loading control. (d) Protein extracts from treatment-naïve BRAF mutant PDX tumours were immunoblotted for the indicated antibodies. (e) Analysis of 356 TCGA melanoma transcriptomes confirming anticorrelation between MITF and RTKs including AXL. $P$ values were computed by a one-sided Wilcoxon ranksum test on the expression levels of $A X L, E G F R$ and PDGFRB between the top quartile (MITF high, $n=89$ ) and bottom quartile (MITF low, $n=89$ ) of MITF expression across the samples.

subtype, we determined MITF expression in NRAS mutant cells by next-generation RNA sequencing. In a set of eight cell lines, we found MITF to be weakly expressed in four, along with the MITF targets MLANA, BCL2 and CDK2 (Fig. 8a). In contrast, the mRNAs encoding AXL, EGFR and PDGFR $\beta$ were predominantly expressed in NRAS mutant cells with low MITF expression. These differences in mRNA levels correlated with the expression levels of their corresponding proteins: an inverse correlation between MITF and RTK expression was also observed in NRAS mutant cells, similar to BRAF mutant cells (Fig. 8b). Consistently, on a phospho-RTK array, AXL and EGFR showed increased phosphorylation in an NRAS mutant MITF ${ }^{\text {endo_lo }}$ cell line (Fig. 8c). In addition, we observed an inverse correlation of MITF and AXL mRNA expression in an independent NRAS mutant and BRAF/NRAS wt cell line panel (Supplementary Fig. 6A) and in NRAS mutant melanoma PDX (Supplementary Fig. 6B).

We also examined the sensitivity of these two classes of cell lines to MEK and ERK inhibitors. In full agreement with our observations for BRAF mutant melanoma, NRAS mutant melanoma cells lacking MITF expression were unresponsive to targeted inhibition of MEK (Fig. 8d) or ERK (Fig. 8e) and resistance correlated with a low MITF/AXL ratio. Combined 
a

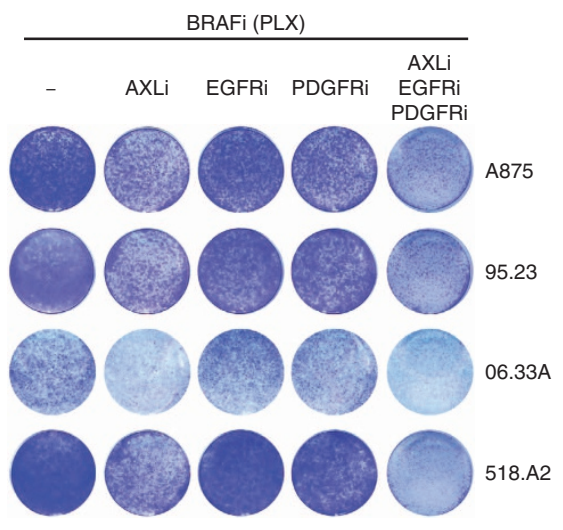

c

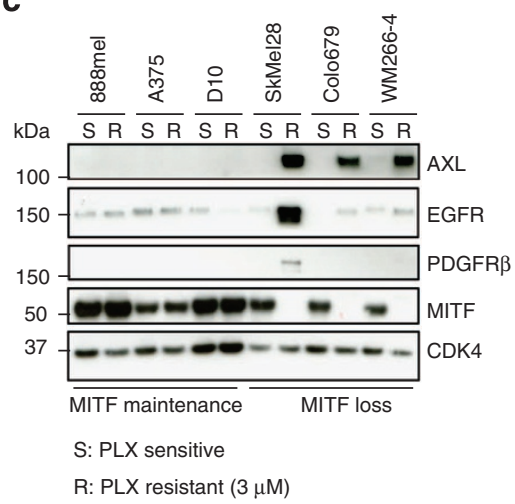

e

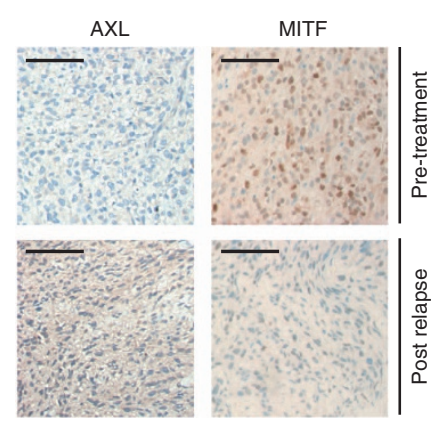

b
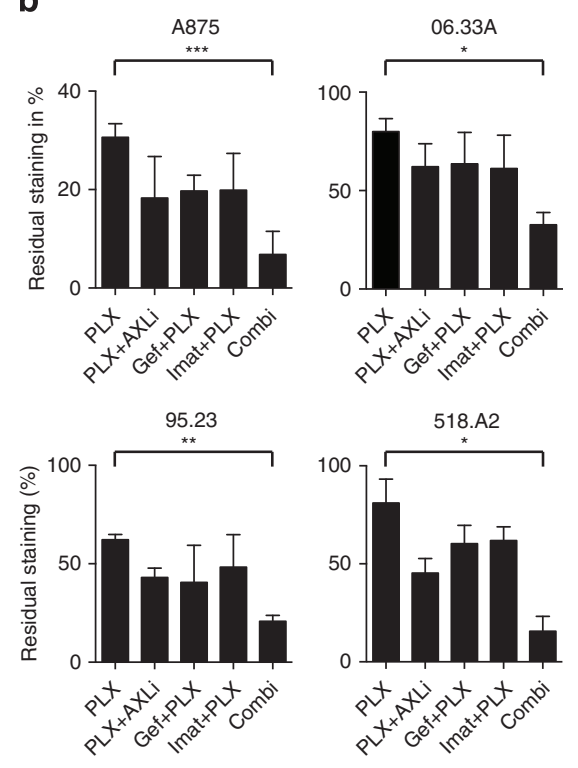

d

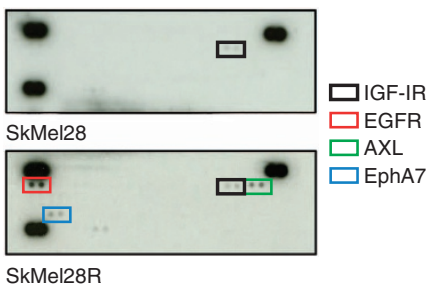

f

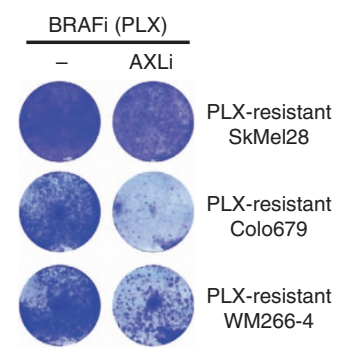

Figure 7 | RTK inhibition increases effect of BRAF inhibition. (a) MITFendo_lo cells were exposed to a combination of RTK inhibitors (AXLi R428 (0.3 $\mu$ M), EGFRi Gefitinib $(2 \mu \mathrm{M})$ and PDGFR $\beta$ I Imatinib $(1 \mu \mathrm{M}))$ and BRAFi PLX4720 $(5 \mu \mathrm{M})$. After 9 days of combined treatment the remaining cells were stained with crystal violet. (b) Quantification of $\mathbf{a}$, comprising three independent experiments. Values were normalized to untreated control (100\%). Error bars represent s.d.; paired $t$-test was performed for statistical analysis, ${ }^{\star} P<0.05,{ }^{\star \star} P<0.01,{ }^{\star \star \star} P<0.001$. (c) PLX4720-resistant melanoma cells and their treatment-naïve counterparts were immunoblotted for AXL, EGFR and PDGFR $\beta$. CDK4 served as a loading control. (d) PLX4720-resistant SkMel28 and their naïve counterparts were blotted on a phospho-RTK array. (e) Matched human melanoma biopsies obtained from a patient before vemurafenib treatment (pre) and after relapse had occurred (post) were stained for MITF and AXL. Scale bars, $100 \mu$ m. (f) AXL-expressing PLX4720-resistant melanoma cells were exposed to $0.3 \mu \mathrm{M}$ of the AXL inhibitor R428 for 9 days and the remaining cells stained with crystal violet.

treatment with ERK and AXL inhibitors of NRAS mutant melanoma cell lines cooperatively decreased viability (Fig. 8f,g). To confirm the specificity of R428, we treated NRAS mutant cells with BMS-777607, another inhibitor targeting AXL, and achieved comparable results to R428 (Supplementary Fig. 6C). In addition, the knockdown of AXL expression by short hairpins in FM6 cells cooperated with ERK inhibition to kill the drugresistant population, in a dose-dependent fashion (Supplementary Fig. 6D). By continuously increasing the concentration, we made a sensitive MITF ${ }^{\text {endo_high }}$ cell line resistant to MEKi (Supplementary Fig. 6E); this cell line was cross-resistant to ERKi. We observed a drastic decrease in MITF and MITF-target gene expression, which was accompanied by sharp increases in AXL and PDGFR expression (Fig. 8h). Thus, similar to BRAF mutant melanoma cells, NRAS mutant melanoma cells harbouring a low MITF/high AXL ratio are resistant to drugs targeting the MAPK pathway, which can be reversed by co-inhibiting AXL.

\section{Discussion}

A large body of evidence has implicated the melanocyte lineage factor MITF not only in melanoma progression but also in drug resistance. For example, MITF is amplified in a subset of human melanomas, ectopically expressed MITF cooperates with mutant 
a

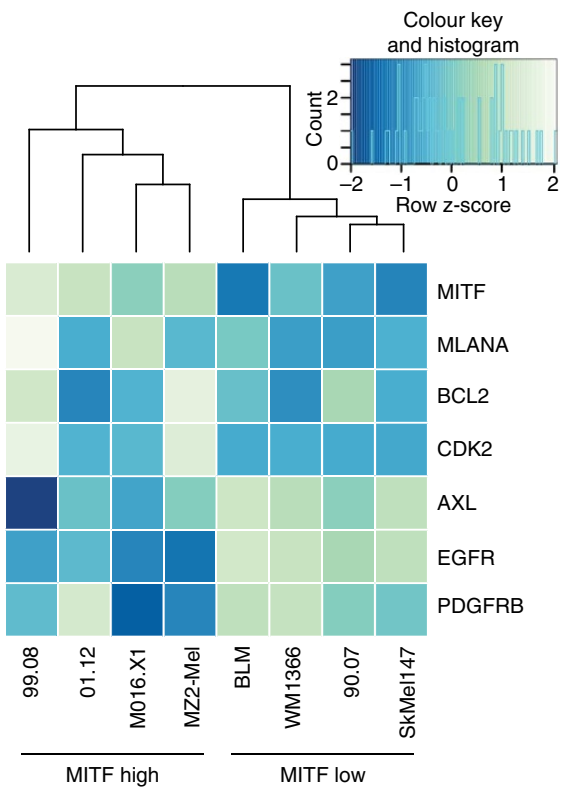

c

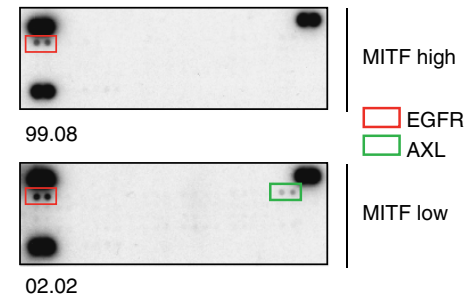

e

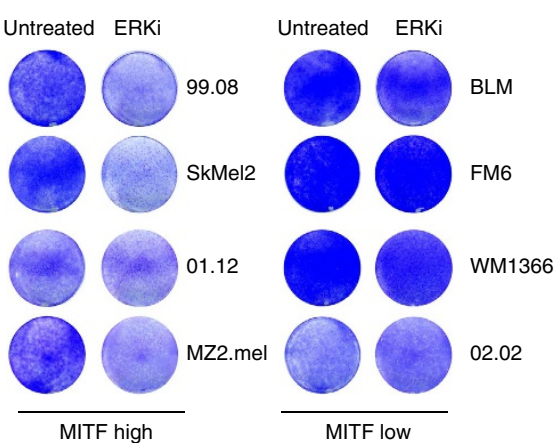

g

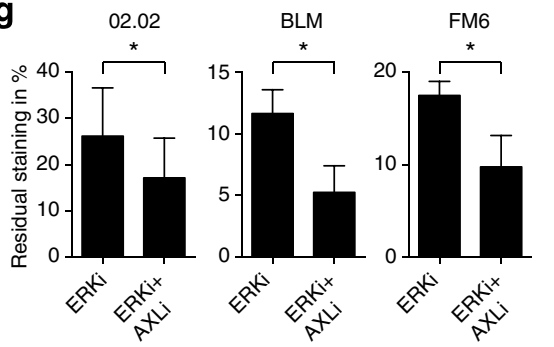

b

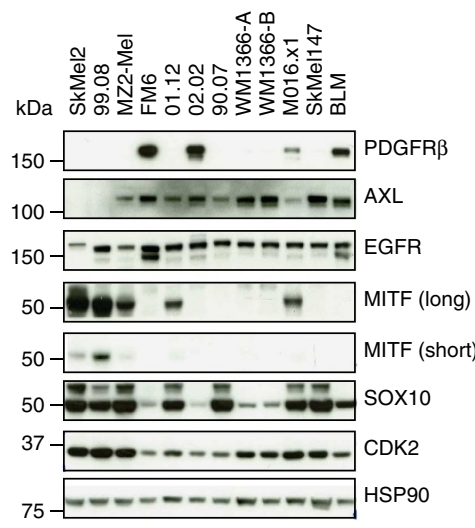

d

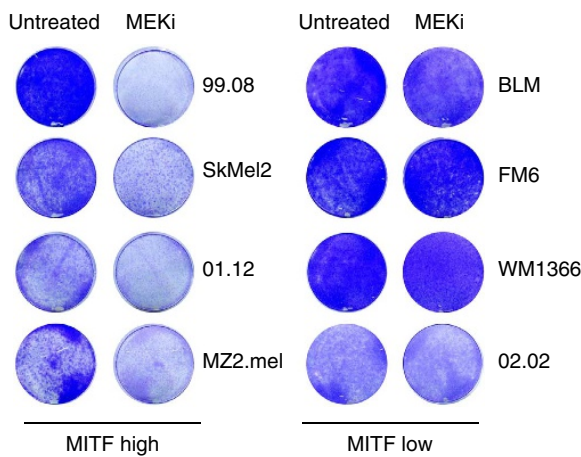

f

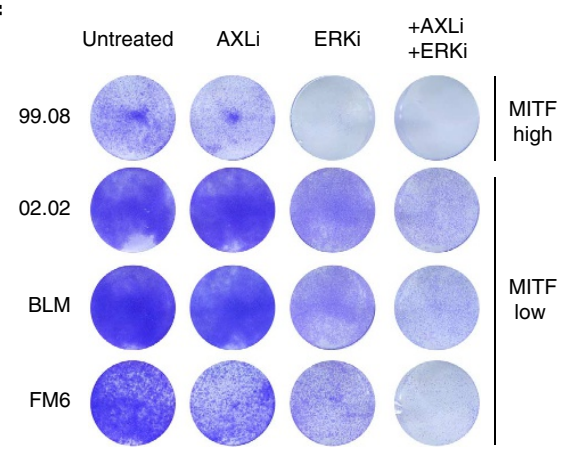

h

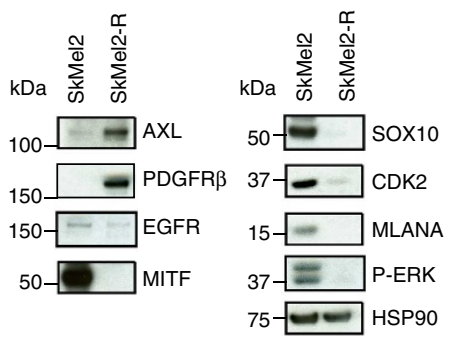

Figure 8 | MITF is a predictive biomarker also in NRAS mutant melanomas. (a) RNA sequencing was performed on four MITFendo_hi and four MITFendo_lo NRAS mutant melanoma cell lines. The genes in the heatmap were selected based on the MITF expression, expression of MITF-target genes and expression of the mRNAs coding for the RTKs AXL, EGFR and PDGFR 3 . (b) Treatment-naïve NRAS mutant melanoma cells were immunoblotted for MITF, RTKs and other proteins as indicated. HSP90 served as a loading control. WM1366-A/B refers to different batches of the same cell line. (c) A phospho-RTK array was performed comparing one NRAS mutant MITFendo_hi and one MITFendo_lo melanoma cell line. (d,e) MITFendo_hi and MITFendo_lo NRAS mutant melanoma cells were plated at low densities and treated with the MEKi trametinib (2.5 nM) (d) or ERKi SCH772984 (0.25 $\mu$ M) (e) or left untreated for 6 days and stained by crystal violet. (f) Melanoma cells were plated at low density and treated with the AXLi R428 (0.5 $\mu$ M), the ERKi $\mathrm{SCH772984}(1 \mu \mathrm{M})$ or in combination. After 6 days of treatment, plates were stained with crystal violet. (g) Quantification of $\mathbf{f}$, based on three biological replicates. Error bars represent s.d.; paired $t$-test was used for statistical analysis, ${ }^{\star} P<0.05$. (h) A MEKi-resistant NRAS mutant melanoma cell line and its treatment-naïve counterpart were blotted for the indicated proteins. HSP90 served as a loading control. 
BRAF in oncogenic transformation and MITF copy gain correlates with chemoresistance ${ }^{22,26}$ Furthermore, a germline $\operatorname{MITF}(\mathrm{E} 318 \mathrm{~K})$ mutation has been suggested to confer increased melanoma susceptibility ${ }^{27-29}$. Consistent with this view, we identified MITF as a locus that was commonly activated in an insertional mutagenesis screen for genes causing resistance to a newly developed ERK inhibitor. Indeed, ectopically expressed MITF caused melanoma cells to become unresponsive to ERK inhibition. In addition, we found MITF levels to be elevated in some PLX4720-resistant cell lines. High-level MITF expression and expression of MITF-target genes have recently been found to play an important role in drug resistance to BRAF inhibition $^{30,31}$, to MEK inhibition ${ }^{23}$ and combined treatment ${ }^{9,12}$. Thus, much of the available evidence points to MITF-driving melanoma progression and contributing to resistance to MAPK pathway inhibition.

When pursuing our initial findings on the role of MITF in mediating resistance to ERK inhibition, however, we observed that also the absence of endogenous MITF expression is beneficial for melanoma cells to survive MAPK pathway inhibition, and that, in fact, endogenous MITF expression was required to adequately respond to the inhibitor. Indeed, very low levels or absence of MITF was invariably associated with intrinsic resistance to several inhibitors and cocktails thereof acting across the MAPK pathway in vitro, in BRAF and NRAS mutant melanoma cells alike. Corroborating these results in the context of relapsing melanoma patients, we observed that in a number of human melanomas that had acquired drug resistance, MITF expression was significantly decreased. In one case, we observed a remarkable divergence, with independent relapsing tumour clones displaying either a strong increase, or a sharp decrease, in MITF expression levels relative to the pre-treatment tumour. Our results suggest that either mechanism can account for the acquisition of a drug-resistant phenotype and merit more comprehensive analyses on the role and exact frequency of MITF deregulation in acquired resistance.

From a cohort of 12 patients, we found MITF to be downregulated in three patients relapsed with resistant melanoma. In comparison, MITF gain was found in five patients. The total number of tumours that gained MITF expression was higher (eight out of 29 versus four out of 29) although one patient in this cohort alone relapsed with five tumours that gained MITF, and we cannot distinguish if those tumours represented independent events or resulted from a pre-established common resistant clone. Despite a modest frequency of MITF loss based on as-yet limited analysis, we speculate that, due to the increased invasiveness and metastatic potential that MITF-low cells display, MITF downregulation might occur more commonly. This would enable the tumour cells to not only become drug resistant but also to disseminate from the primary tumour. They may regain MITF expression again while forming a secondary tumour, similar to the reversion of EMT downstream in the metastatic cascade, but this needs to be investigated further.

Our results suggest that in the context of acquired resistance, at least two distinct classes of melanomas exist: the first maintains or increases MITF expression (denoted here as MITF ${ }^{\text {acq_maint }}$ cells); the second class (MITF ${ }^{\text {acq_loss }}$ cells), comprising at least half of the cell line panels we studied, sharply downregulates MITF expression on acquiring the resistance trait. Characterizing MITF $^{\text {acq_loss }}$ and MITF acq_maint PLX4720-resistant cells, we found that loss of MITF correlated with increased cross-resistance towards MAPK pathway inhibitors. This suggests that MITF loss marks a population of cells that are proliferating and surviving independently of the MAPK pathway. Furthermore, these cells showed increased invasive capacity associated with an EMT-like signature, as marked by a decrease in E-Cadherin. Dial mRNA followed the MITF expression pattern in acquired resistance cells, potentially facilitating Rock1-dependent invasiveness ${ }^{19}$. Invasiveness was also associated and partly caused by increased expression of the AP1 family transcription factor Fra-1, which we recently demonstrated to drive a metastatic programme in human breast cancer ${ }^{25}$. These findings are in line with, and extend, a model that has been proposed previously ${ }^{32,33}$. It describes a dynamic phenotypic switch between proliferative and invasive states that melanoma cells can undergo to gain invasive properties and metastasize. This idea is consistent with a recent report by Garraway and co-workers ${ }^{34}$, describing a melanoma drug response as a function of transcriptional cell states linked to MITF and NF- $\kappa B$. Elaborating on this concept, it has been reported that subpopulations of slowly cycling melanoma cells exist, which are marked by the H3K4 demethylase ${ }^{35}$ and low MITF levels ${ }^{36}$. The switching phenomenon can be accompanied by loss of MITF and dedifferentiation of primary melanoma cells as marked by loss of MelanA ${ }^{37}$. Furthermore, it has been reported recently that the transcription factors Zeb1 and Zeb2 not only regulate EMT but are expressed in opposed directions in melanocytes, thereby controlling MITF expression and differentiation $^{38}$. Loss of Zeb2 results in dedifferentiation, accompanied by loss of MITF and upregulation of Zeb1, further supporting the idea that acquired resistant cells can lose MITF and dedifferentiate. Our data suggest that therapy-resistant melanoma cells undergo a similar phenotypic switch and dedifferentiation as a function of MITF loss of, marking an invasive subpopulation of resistant cancer cells.

In treatment-naïve MITF-low cells, we found a remarkable inverse correlation between the absence of MITF and increased expression levels of several RTKs, predominantly EGFR, PDGFR $\beta$ and AXL. Likewise, in acquired resistance, we detected a striking inverse correlation between the loss of MITF and gain of expression of the aforementioned RTKs, particularly AXL. These observations suggest that the expression of these RTKs protects melanoma cells from targeted therapy and that their expression is, either directly or indirectly, negatively regulated by MITF. Recently, SOX10 was identified as a negative regulator of EGFR expression in melanoma, suggesting that loss of SOX10 leads to an increase in EGFR expression leading to acquired resistance ${ }^{39}$. SOX10 can directly transactivate the MITF promoter $^{40}$ and cooperates with MITF in activating further downstream targets ${ }^{41}$. However, despite a correlation between SOX10 and MITF expression, we find that absence of MITF and expression of RTKs are not strongly dependent on the absence of SOX10 in treatment-naïve melanoma cells, certainly not in those harbouring NRAS mutation, nor in cells that have acquired resistance. These data suggest that MITF is a central determinant of RTK expression and drug resistance in melanoma.

Given the resistance emerging commonly to treatment with single inhibitors of the MAPK pathway, experimental combinatorial therapies have been launched. But early clinical data on BRAF mutant melanoma patients treated with a combinatorial approach of BRAF and MEK inhibitors (dabrafenib + trametinib) indicates that unfortunately, even in that setting eventually most patients become resistant to the treatment ${ }^{13,42}$. Thus, addition of an alternative or third compound prolonging clinical response is highly needed. Our results predict that MITF-low melanoma patients, whether harbouring a BRAF or NRAS mutation, may benefit from additional AXL inhibition. We show that single inhibition of AXL strongly decreases viability in two out of three PLX4720-resistant cells even in the absence of the BRAF inhibitor, whereas AXL alone does not affect treatment-naïve cells. In some cases, the pattern is more complex, because in addition to AXL, other RTKs are upregulated. For instance, in PLX4720-resistant SkMel28 cells, we observe in addition to AXL 
also the upregulation of EGFR and PDGFR $\beta$, likely explaining why AXL inhibition alone was insufficiently cytotoxic for this cell line. These results underscore the strong resistance phenotype of MITF-low melanomas. We propose that the loss of MITF expression marks melanomas that display early resistance to a broad range of targeted inhibitors and cocktails thereof.

An inverse correlation between the expressions of MITF and AXL has been reported previously $9,43,44$. AXL is a tyrosine receptor kinase that is overexpressed in several human cancers including ocular melanoma ${ }^{45-47}$. Interestingly, in addition to its implication in promoting tumour growth, AXL has been reported to induce EMT and metastasis ${ }^{48}$. AXL expression is directly transcriptionally regulated by the Fra- 1 transcription factor in bladder cancer ${ }^{49}$. In cells that have acquired resistance, we find coincident increases in Fra-1 and AXL expression and a stronger invasive phenotype, which are accompanied by a decrease in MITF. These results suggest a model for MITF-dependent repression (whether directly or indirectly) of several RTKs.

In conclusion, there is a wide body of evidence speaking to MITF driving both melanoma progression and drug resistance, which is consistent with our finding that the overexpression of MITF renders melanomas less susceptible to targeted inhibitors. But we show here that also instead, the loss of MITF marks a melanoma class that is at least as drug resistant, and to a broader range of MAPK pathway and other targeted inhibitors, as cells with amplified MITF. This is associated with high expression (mostly by direct and indirect derepression) and activation of several RTKs. Our results therefore demonstrate that a low MITF/AXL ratio predicts resistance to multiple targeted drugs impacting on the MAPK pathway and RTKs. They also provide a rationale for clinically exploring AXL inhibitors to combat resistance in MITF-low/AXL high, BRAF and NRAS mutant melanomas.

\begin{abstract}
Methods
Validation-based insertional mutagenesis screen. The insertional mutagenesis screen was performed as previously described ${ }^{24}$. In brief, an intermediately sensitive melanoma cell line of low passage (04.07) was infected separately with the three different VBIM constructs (SD1-3) in three 48-well plates (10,000 cells per well). Two days after infection, the cells were transferred to six-well plates. The next day they were exposed to $1 \mu \mathrm{M}$ SCH772984 for 3 weeks until single colonies had formed. These were picked and cultured individually in the presence of the drug. For identification of insertions, DNA was isolated using DNAzol reagent (Invitrogen). Splinkerette PCR was used according to the published protocol ${ }^{19,24}$ and subsequently Sanger sequencing was applied on the amplified products.
\end{abstract}

Vectors. MITF-M was amplified from human melanocyte cDNA and cloned into pcDHpuro using EcoR1 and Not1 restriction sites.

Forward primer: $5^{\prime}$-GGGGAATTCATGGATGCTGGAAATGCTAGAATATA ATCACTATCAGG- $3^{\prime}$

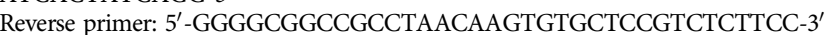
FRA-1 and AXL hairpins were from the TRC library.

FRA-1: \#41: TRCN0000019541, \#42: TRCN0000019542

AXL: \#572: TRCN0000000572, \#573: TRCN0000000573,

\#575: TRCN0000000575.

qRT-PCR and primers. RNA was isolated using Trizol (Ambion), following standard protocol. Reverse transcription was performed with oligo-dT primers. qRT-PCR-primers were designed using Primer3 software. qRT-PCR was performed with SYBR green master mix (Applied Biosystems, Warrington, UK) on a StepOnePlus Real-Time PCR system (Applied Biosystems). RNA levels were normalized against $\beta$-ACTIN, GAPDH, TUB4A or RPL13.

The following primers were used:

MITF forward: $5^{\prime}$-CAGGCATGAACACACATTCAC- $3^{\prime}$

MITF reverse: $5^{\prime}$-TCCATCAAGCCCAAGATTTC-3'

MITF- $M$ forward: $5^{\prime}$-GGAGCTCACAGCGTGTATTTTTCC- $3^{\prime}$

MITF- $M$ reverse: $5^{\prime}$-GACTTGGGAATCAAAGTACCTAGTTC- ${ }^{\prime}$

ACTINB forward: 5'-CCAACCGCGAGAAGATGA- $3^{\prime}$

ACTINB reverse: 5'-CCAGAGGCGTACAGGATAG- $3^{\prime}$

RPL13 forward: $5^{\prime}$-GAGACAGTTCTGCTGAAGAACTGAA- ${ }^{\prime}$

RPL13 reverse: $5^{\prime}$-TCCGGACGGGCATGAC- $3^{\prime}$
$Z E B 1$ forward: $5^{\prime}$-TGCACTGAGTGTGGAAAAGC- $3^{\prime}$ $Z E B 1$ reverse: $5^{\prime}$-TTGCAGTTTGGGCATTCATA- $3^{\prime}$ ZEB2 forward: $5^{\prime}$-AGTACCAGCGGAAACAAGGA- $3^{\prime}$ ZEB2 reverse: $5^{\prime}$-TTTTTGCGAGACAGACAGGA-3' CDH1 forward: $5^{\prime}$-GGCCAGGAAATCACATCCTA-3' CDH1 reverse: $5^{\prime}$-AGCCAGTTGGCAGTGTCTCT- ${ }^{\prime}$ $\mathrm{CDH} 2$ forward: $5^{\prime}$-GGACCGAGAATCACCAAATG-3' $\mathrm{CDH} 2$ reverse: $5^{\prime}$-AGATCTGCAGCGTTCCTGTT-3' TUBA4A forward: $5^{\prime}$-GACAGCTCTTCCACCCAGAG- $3^{\prime}$ TUBA4A reverse: $5^{\prime}$-TGAAGTCCTGTGCACTGGTC- $3^{\prime}$ GAPDH forward: $5^{\prime}$-CAATGACCCCTTCATTGACC- ${ }^{\prime}$ GAPDH reverse: 5'-GACAAGCTTCCCGTTCTCAG-3'.

Inhibitors and solvents. MEK inhibitor GSK1120212/trametinib, BRAF inhibitors PLX4720, PLX4032/vemurafenib and GSK211436/dabrafenib, EGFR inhibitor gefitinib, c-Kit/PDGFR inhibitor imatinib/Gleevec and AXL inhibitor BMS-777607 were all purchased from Selleck Chemicals (Houston, TX, USA). ERK inhibitor SCH772984 was provided by Merck \& Co, Whitehouse Station, NJ, USA (under an MTA). AXL inhibitor R428 was from Axon Medchem (Groningen, the Netherlands), the metabolic poison phenyl arsine oxide (PAO) and solvent dimethylsulfoxide were from Sigma-Aldrich (St Louis, MO, USA). All drugs were reconstituted in $100 \%$ dimethylsulfoxide to a final concentration of $10-20 \mathrm{mM}$.

Cell lines, cell culture conditions and treatments. Melanoma cell lines and HEK293T cells were cultured in DMEM with fetal bovine serum (Sigma), $2 \mathrm{mM}$ glutamine, $100 \mathrm{U} \mathrm{ml}^{-1}$ penicillin and $0.1 \mathrm{mg} \mathrm{ml}^{-1}$ streptomycin (all Gibco) under standard conditions. HEK293T cells were used for virus production for MITF overexpression. In brief, HEK293T cells were transfected with the plasmid of interest and the helper plasmids (pMDLglpRRE, pHCMV-G and pRSVrev). Viral supernatant was either snap frozen or immediately used for infection. Infected melanoma cells were selected with puromycin (Sigma). To generate BRAFi- or ERKi-resistant cells, cells were exposed to increasing concentrations of PLX4720 (up to $3 \mu \mathrm{M}$ ) or SCH772984 (up to $1 \mu \mathrm{M}$ ) and resistance was confirmed by measuring cell viability under treatment. All drug-resistant cell lines were permanently cultured under $3 \mu \mathrm{M}$ of PLX4720, $1 \mu \mathrm{M}$ vemurafenib, $1 \mu \mathrm{M} \mathrm{SCH772984} \mathrm{or} 0.1 \mu \mathrm{M}$ GSK211436.

For dose-response curves and viability assays, cells were plated and equal concentration of inhibitors was added 1 day after seeding. For short-term viability assays (dose-response curves), cells were plated in a 96-well format and drugs were diluted with the HP D300 Digital Dispenser (Tecan, Giessen, Germany). After 3 to 5 days of incubation, the medium was replaced by a dilution of CellTiter Blue reagent or CellTiter-Glo Luminescent Cell Viability Assay (Promega, Madison, WI) in medium. Two hours later, luminescence was measured by the Infinite M200 microplate reader (Tecan). Long-term viability assays were performed in 6- or 12well plate format. Inhibitor solutions were replaced every 2 to 3 days and plates were stained with crystal violet after 5 to 9 days of treatment (as indicated). For protein, DNA and RNA analysis, cells were treated on 10-cm dishes and snap frozen after harvesting.

Patient-derived xenografts. To generate PDX, human melanoma fragments were collected after surgical resection and transplanted subcutaneously into NSG mice. PDX were maintained in mice for subsequent passages. For this, cells from the first passage PDX (X1) were dissociated by $200 \mathrm{U} \mathrm{ml}^{-1}$ collagenase $/ 1 \mu \mathrm{g} \mathrm{ml}^{-1}$ DNAse incubation for $20 \mathrm{~min}$ at $37^{\circ} \mathrm{C}$, filtered through a $70-\mu \mathrm{m}$ cell strainer, washed, resuspended in matrigel and subcutaneously injected into NSG mice $\left(5 \times 10^{5}\right.$ per flank). Alternatively, PDX-derived cell lines (M016) were generated by dissociating tumours and cultured under standard conditions.

Invasion assay and Phospho-RTK array. To examine the invasive potential 200,000 freshly trypsinized cells were seeded on matrigel-coated chambers (BD Biosciences) in serum-free medium. The lower compartment contained medium supplemented with $9 \%$ fetal bovine serum. After overnight incubation, non-invasive cells were removed from the chamber and invaded cells were stained with crystal violet. Pictures of invaded cells were taken on an Axio Vert A1 microscope (Zeiss).

Phospho-RTK arrays were performed using the human Phospho-RTK Array from RD Systems (Minneapolis, USA). All steps were performed according to the manufacturer's protocol.

Immunohistochemistry. EAF-fixed tumour samples were embedded in paraffin and stained with haematoxilin/eosin according to standard procedures.

Western blotting and antibodies. Cell pellets were lysed in RIPA buffer $(50 \mathrm{mM}$ TRIS pH 8.0, $150 \mathrm{mM} \mathrm{NaCl}, 1 \%$ Nonidet P40, $0.5 \%$ sodium deoxycholate, $0.1 \%$ SDS) supplemented with complete protease inhibitor cocktail (Roche Applied Science) and phosphatase inhibitors $10 \mathrm{mM} \mathrm{NaF}, 1 \mathrm{mM}$ Na3VO4, $1 \mathrm{mM}$ sodium pyrophosphate, $10 \mathrm{mM}$ beta-glycerophosphate. Protein concentration was determined with the Protein Assay (Bio-Rad). Western blotting was performed with 
standard techniques using 4-12\% Bis-Tris polyacrylamide-SDS gels (NuPAGE, Life Technologies) and nitrocellulose membranes (Whatman, GE Healthcare). Blots were blocked in $4 \%$ milk in PBS plus $0.2 \%$ Tween 100 and incubated with primary antibodies: Phospho-AKT (1:1,000, sc-7985-R), AXL (1:500, sc-1096), BCL2 (1:1,000, sc-492), CDK2 (1:1,000, sc-163), CDK4 (1:1,000, sc-601), EGFR (1:500, sc-03), FRA-1 (1:1,000, sc-605X) and HSP90 (1:5,000, sc-7947) were purchased from Santa Cruz; E-Cadherin (1:1,000, 610181) and N-Cadherin $(1: 1,000,610920)$ from BD Biosciences; MAP kinase p44/42 Thy202/Tyr204 (1:1,000, 9106), MEK 1/ 2 phospho $(1: 1,000,9154 \mathrm{~S})$, PARP $(1: 1,000,9542)$ and PDGFR $\beta(1: 1,000,3169 \mathrm{~S})$ from Cell Signaling; MLANA (1:5,000, MS 716P0) and MITF (1:1,000, MS-771P1) from Neomarkers; MITF (1:1,000, ab12039) and Sox10 (1:1,000, ab-17732) from Abcam; RSK1 Thr359/Ser363 from Millipore (1:1,000); FLAG (1:1,000, F3165) and $\alpha$-Tubulin (1:5,000, T9026) from Sigma. The following secondary antibodies were used: swine anti-goat IgG (1:2,000, ALI0404, Biosource), goat antirabbit peroxidase conjugate $(1: 5,000, \mathrm{G} 21234)$ and goat anti-mouse (1:5,000, G21040), both purchased from Invitrogen. Western blots were incubated in a 1:1 dilution of solution $1(0.1 \mathrm{M}$ Tris $\mathrm{pH} 8,2.5 \mathrm{mM}$ luminol, $0.4 \mathrm{mM}$ p-Coumaric acid, all Sigma) and solution2 (0.1 M Tris $\mathrm{pH} 8,30 \% \mathrm{H}_{2} \mathrm{O}_{2}$, all Sigma) and chemiluminescent signal was visualized using high performance autoradiography films (Hyperfilm MP, Amersham).

RNA-seq analysis. Illumina 50 bp paired-end RNA-seq data was collected on a panel of 28 melanoma cell lines. Read mapping was performed using TopHat version 2.0.9 with the NCBI Build 37 reference genome ${ }^{50}$. Read counts per gene were quantified using HTSeq version 0.5.4. Counts were adjusted for gene length and GC content and quantile normalized using the CQN R package to obtain gene level offsets. Read counts were fitted to a generalized linear model with offsets for the final normalization step using the DESeq2 $\mathrm{R}$ package ${ }^{51}$. Pearson correlation coefficients between genes for all samples were calculated using $\mathrm{R}^{52-54}$.

Illumina $90 \mathrm{bp}$ paired-end RNA-seq data was collected on 14 melanoma cell lines. Read mapping was performed using the same approach as for the melanoma cell line panel as described above. Read counts were transformed by applying a variance stabilization with DESeq (1.12.1). In DESeq the dispersion estimate estimateDispersions had parameters: method $=$ 'per-condition' and fitType $=$ 'local' and for null model evaluation with no replicates method= 'blind', and sharingMode = 'fit-only'. Heatmaps were generated with gplots (2.12.1) as available through Bioconductor. Analysis was performed, and plots were made using the statistical programming language $\mathrm{R}$ (v 3.0.2).

Level 3 RNA sequencing expression values in FPKM for MITF, AXL, EGFR and PDGFRB were downloaded from the TCGA website (http://cancergenome.nih.gov/) for the Skin Cutaneous Melanoma [SKCM] samples. Differential expression was computed by one-sided wilcoxon rank test on the expression distribution of the gene in the MITF-high group (tumour samples in top quartile of MITF expression) and MITF-low group (samples in the bottom quartile of MITF expression). The RNA-seq data are available under accession number GSE61544 and GSE62526.

\section{References}

1. Davies, H. et al. Mutations of the BRAF gene in human cancer. Nature 417, 949-954 (2002).

2. Chapman, P. B. et al. Improved survival with vemurafenib in melanoma with BRAF V600E mutation. New Engl. J. Med. 364, 2507-2516 (2011).

3. Tsai, J. et al. Discovery of a selective inhibitor of oncogenic B-Raf kinase with potent antimelanoma activity. Proc. Natl Acad. Sci. USA 105, 3041-3046 (2008).

4. Bollag, G. et al. Clinical efficacy of a RAF inhibitor needs broad target blockade in BRAF-mutant melanoma. Nature 467, 596-599 (2010).

5. Wagle, N. et al. Dissecting therapeutic resistance to RAF inhibition in melanoma by tumor genomic profiling. J. Clin. Oncol. 29, 3085-3096 (2011).

6. Nazarian, R. et al. Melanomas acquire resistance to B-RAF(V600E) inhibition by RTK or N-RAS upregulation. Nature 468, 973-977 (2010).

7. Emery, C. M. et al. MEK1 mutations confer resistance to MEK and B-RAF inhibition. Proc. Natl Acad. Sci. USA 106, 20411-20416 (2009).

8. Poulikakos, P. I. et al. RAF inhibitor resistance is mediated by dimerization of aberrantly spliced BRAF(V600E). Nature 480, 387-390 (2011).

9. Johannessen, C. M. et al. A melanocyte lineage program confers resistance to MAP kinase pathway inhibition. Nature 504, 138-142 (2013).

10. Villanueva, J. et al. Acquired resistance to BRAF inhibitors mediated by a RAF kinase switch in melanoma can be overcome by cotargeting MEK and IGF-1R/ PI3K. Cancer Cell 18, 683-695 (2010).

11. Straussman, R. et al. Tumour micro-environment elicits innate resistance to RAF inhibitors through HGF secretion. Nature 487, 500-504 (2012).

12. Shi, H. et al. Acquired resistance and clonal evolution in melanoma during BRAF inhibitor therapy. Cancer Discov. 4, 80-93 (2014).

13. Shi, H. et al. A novel AKT1 mutant amplifies an adaptive melanoma response to BRAF inhibition. Cancer Discov. 4, 69-79 (2014).

14. Hodi, F. S. et al. Improved survival with ipilimumab in patients with metastatic melanoma. New Engl. J. Med. 363, 711-723 (2010).

15. Topalian, S. L. et al. Safety, activity, and immune correlates of anti-PD-1 antibody in cancer. New Engl. J. Med. 366, 2443-2454 (2012).
16. Flaherty, K. T. et al. Improved survival with MEK inhibition in BRAF-mutated melanoma. New Engl. J. Med. 367, 107-114 (2012).

17. Morris, E. J. et al. Discovery of a novel ERK inhibitor with activity in models of acquired resistance to BRAF and MEK inhibitors. Cancer Discov. 3, 742-750 (2013).

18. Hodgkinson, C. A. et al. Mutations at the mouse microphthalmia locus are associated with defects in a gene encoding a novel basic-helix-loop-helix-zipper protein. Cell 74, 4622-4626 (1993).

19. Carreira, S. et al. Mitf regulation of Dial controls melanoma proliferation and invasiveness. Genes Dev. 20, 3426-3439 (2006).

20. Carreira, S. et al. Mitf cooperates with Rb1 and activates p21Cip1 expression to regulate cell cycle progression. Nature 433, 764-769 (2005).

21. Loercher, A. E., Tank, E. M. H., Delston, R. B. \& Harbour, J. W. MITF links differentiation with cell cycle arrest in melanocytes by transcriptional activation of INK4A. J. Cell. Biol. 168, 35-40 (2005).

22. Garraway, L. A. et al. Integrative genomic analyses identify MITF as a lineage survival oncogene amplified in malignant melanoma. Nature 436, 117-122 (2005).

23. Smith, M. P. et al. Effect of SMURF2 targeting on susceptibility to MEK inhibitors in melanoma. J. Natl Cancer Inst. 105, 33-46 (2013).

24. Lu, T. et al. Validation-based insertional mutagenesis identifies lysine demethylase FBXL11 as a negative regulator of NFkappaB. Proc. Natl Acad. Sci. USA 106, 16339-16344 (2009).

25. Desmet, C. J. et al. Identification of a pharmacologically tractable Fra-1/ ADORA2B axis promoting breast cancer metastasis. Proc. Natl Acad. Sci. 110, 5139-5144 (2013).

26. Hertzman Johansson, C. et al. Association of MITF and other melanosomerelated proteins with chemoresistance in melanoma tumors and cell lines. Melanoma Res. 23, 360-365 (2013).

27. Bertolotto, C. et al. A SUMOylation-defective MITF germline mutation predisposes to melanoma and renal carcinoma. Nature 480, 94-98 (2011).

28. Yokoyama, S. et al. A novel recurrent mutation in MITF predisposes to familial and sporadic melanoma. Nature 480, 99-103 (2011).

29. Tsao, H., Chin, L., Garraway, L. A. \& Fisher, D. E. Melanoma: from mutations to medicine. Genes Dev. 26, 1131-1155 (2012).

30. Haq, R. et al. BCL2A1 is a lineage-specific antiapoptotic melanoma oncogene that confers resistance to BRAF inhibition. Proc. Natl Acad. Sci. USA 110, 4321-4326 (2013).

31. Van Allen, E. M. et al. The genetic landscape of clinical resistance to RAF inhibition in metastatic melanoma. Cancer Discov. 4, 94-109 (2014).

32. Hoek, K. S. \& Goding, C. R. Cancer stem cells versus phenotype-switching in melanoma. Pigment Cell Melanoma Res. 23, 746-759 (2010).

33. Hoek, K. S. et al. In vivo switching of human melanoma cells between proliferative and invasive states. Cancer Res. 68, 650-656 (2008).

34. Konieczkowski, D. J. et al. A melanoma cell state distinction influences sensitivity to MAPK pathway inhibitors. Cancer Discov. 4, 816-827 (2014).

35. Roesch, A. et al. A temporarily distinct subpopulation of slow-cycling melanoma cells is required for continuous tumor growth. Cell 141, 583-594 (2010).

36. Cheli, Y. et al. Mitf is the key molecular switch between mouse or human melanoma initiating cells and their differentiated progeny. Oncogene 30, 2307-2318 (2011).

37. Steingrímsson, E., Copeland, N. G. \& Jenkins, N. A. Melanocytes and the microphthalmia transcription factor network. Annu. Rev. Genet. 38, 365-411 (2004).

38. Denecker, G. et al. Identification of a ZEB2-MITF-ZEB1 transcriptional network that controls melanogenesis and melanoma progression. Cell Death Differ. 21, 1250-1261 (2014).

39. Sun, C. et al. Reversible and adaptive resistance to BRAF(V600E) inhibition in melanoma. Nature 508, 118-122 (2014).

40. Bondurand, N. et al. Interaction among SOX10, PAX3 and MITF, three genes altered in Waardenburg syndrome. Hum. Mol. Genet. 9, 1907-1917 (2000).

41. Murisier, F., Guichard, S. \& Beermann, F. The tyrosinase enhancer is activated by Sox10 and Mitf in mouse melanocytes. Pigment Cell Res. 20, 173-184 (2007)

42. Wagle, N. et al. MAP kinase pathway alterations in BRAF-mutant melanoma patients with acquired resistance to combined RAF/MEK inhibition. Cancer Discov. 4, 61-68 (2014).

43. Sensi, M. et al. Human cutaneous melanomas lacking MITF and melanocyte differentiation antigens express a functional Axl receptor kinase. J. Invest. Dermatol. 131, 2448-2457 (2011).

44. Ohanna, M. et al. Secretome from senescent melanoma engages the STAT3 pathway to favor reprogramming of naive melanoma towards a tumorinitiating cell phenotype. Oncotarget 4, 2212-2224 (2013).

45. van Ginkel, P. R. et al. Expression of the receptor tyrosine kinase Axl promotes ocular melanoma cell survival. Cancer Res. 64, 128-134 (2004). 
46. Shieh, Y.-S. et al. Expression of axl in lung adenocarcinoma and correlation with tumor progression. Neoplasia 7, 1058-1064 (2005).

47. Zhang, Y. X. et al. AXL is a potential target for therapeutic intervention in breast cancer progression. Cancer Res. 68, 1905-1915 (2008).

48. Gjerdrum, C. et al. Axl is an essential epithelial-to-mesenchymal transitioninduced regulator of breast cancer metastasis and patient survival. Proc. Natl Acad. Sci. USA 107, 1124-1129 (2010).

49. Sayan, A. E. et al. Fra-1 controls motility of bladder cancer cells via transcriptional upregulation of the receptor tyrosine kinase AXL. Oncogene 31, 1493-1503 (2012).

50. Trapnell, C., Pachter, L. \& Salzberg, S. L. TopHat: discovering splice junctions with RNA-Seq. Bioinformatics 25, 1105-1111 (2009).

51. Anders, S. \& Huber, W. Differential expression analysis for sequence count data. Genome Biol. 11, R106 (2010).

52. Kim, D. et al. TopHat2: accurate alignment of transcriptomes in the presence of insertions, deletions and gene fusions. Genome Biol. 14, R36 (2013).

53. Trapnell, C. et al. Differential analysis of gene regulation at transcript resolution with RNA-seq. Nat. Biotechnol. 31, 46-53 (2013).

54. Roberts, A., Trapnell, C., Donaghey, J., Rinn, J. L. \& Pachter, L. Improving RNA-Seq expression estimates by correcting for fragment bias. Genome Biol. 12, R22 (2011).

\section{Acknowledgements}

We thank Erwin Klein and Menno Hoogeveen for experimental help, Guus Heynen and Rene Bernards for help with setting up the VBIM system, Els Verdegaal and Sjoerd van der Burg (Leiden University Medical Center) for providing low-passage melanoma cell lines, and all members of the Peeper laboratory for their valuable input. We thank Merck for financial support and for providing ERK inhibitor SCH772984. This work was supported by an EMBO long-term fellowship (J.M.), NIH grant P01 CA168585 (A.R., R.S.L. and T.G.G.), a Research Scholar Award from the American Cancer Society
(RSG-12-257-01-TBE) and an Established Investigator Award from the Melanoma Research Alliance (20120279), by NIH/National Center for Advancing Translational Science (NCATS) UCLA CTSI Grant Number UL1TR000124 (T.G.G.), a Queen Wilhelmina award and a grant from the Dutch Cancer Society (KWF) (P.A.P., K.K., P.D.M.C.-S. and D.S.P.) and an ERC Synergy grant (J.M., P.A.P., O.K. and D.S.P.).

\section{Author contributions}

J.M. and D.S.P. designed the study, analysed the data and wrote the manuscript; J.M. performed the experiments; O.K. and W.H. performed the bioinformatic analyses; C.S. J.T., L.R., X.K. and P.A.P. engineered and analysed the cell line panels; P.D.M.C.-S. helped with immunohistochemical staining; M.H.G.F., K.K., C.B. and J.H. helped with characterizing melanoma samples; C.R.G. shared unpublished results and gave critical input; U.M.D. provided matched patient biopsies; T.G.G., A.R., R.S.L. and D.S.P. supervised the study.

\section{Additional information}

Supplementary Information accompanies this paper at http://www.nature.com/ naturecommunications

Competing financial interests: A patent related to the findings reported here has been filed with J.M. and D.S.P. as inventors. The remaining authors declare no competing financial interests.

Reprints and permission information is available online at http://npg.nature.com/ reprintsandpermissions/

How to cite this article: Müller, J. et al. Low MITF/AXL ratio predicts early resistance to multiple targeted drugs in melanoma. Nat. Commun. 5:5712 doi: 10.1038/ncomms6712 (2014). 